\title{
Interferometric observations of warm deuterated methanol in the inner regions of low-mass protostars ${ }^{\star}$
}

\author{
V. Taquet ${ }^{1}$, E. Bianchi' ${ }^{2,1}$, C. Codella ${ }^{1,2}$, M. V. Persson ${ }^{3}$, C. Ceccarelli ${ }^{2}$, S. Cabrit ${ }^{4}$, J. K. Jørgensen ${ }^{5}$, C. Kahane ${ }^{2}$, \\ A. López-Sepulcre ${ }^{2,6}$, and R. Neri ${ }^{6}$ \\ ${ }^{1}$ INAF, Osservatorio Astrofisico di Arcetri, Largo E. Fermi 5, 50125 Firenze, Italy \\ e-mail: taquet@arcetri.astro.it \\ 2 IPAG, Université Grenoble Alpes, CNRS, 38000 Grenoble, France \\ ${ }^{3}$ Department of Space, Earth, and Environment, Chalmers University of Technology, Onsala Space Observatory, 43992 Onsala, \\ Sweden \\ ${ }^{4}$ LERMA, Observatoire de Paris, PSL Research University, CNRS, Sorbonne Université, UPMC Université Paris 06, 75014 Paris, \\ France \\ ${ }^{5}$ Niels Bohr Institute, University of Copenhagen, Øster Voldgade 5-7, 1350 Copenhagen K., Denmark \\ ${ }^{6}$ Institut de Radioastronomie Millimétrique, 38406 Saint-Martin d'Hères, France
}

Received 7 June 2019 / Accepted 19 September 2019

\begin{abstract}
Methanol is a key species in astrochemistry because it is the most abundant organic molecule in the interstellar medium and is thought to be the mother molecule of many complex organic species. Estimating the deuteration of methanol around young protostars is of crucial importance because it highly depends on its formation mechanisms and the physical conditions during its moment of formation. We analyse several dozen transitions from deuterated methanol isotopologues coming from various existing observational datasets obtained with the IRAM-PdBI and ALMA sub-millimeter interferometers to estimate the methanol deuteration surrounding three low-mass protostars on Solar System scales. A population diagram analysis allows us to derive a $\left[\mathrm{CH}_{2} \mathrm{DOH}\right] /\left[\mathrm{CH}_{3} \mathrm{OH}\right]$ abundance ratio of 3-6\% and a $\left[\mathrm{CH}_{3} \mathrm{OD}\right] /\left[\mathrm{CH}_{3} \mathrm{OH}\right]$ ratio of $0.4-1.6 \%$ in the warm inner $(\leq 100-200 \mathrm{AU})$ protostellar regions. These values are typically ten times lower than those derived with previous single-dish observations towards these sources, but they are one to two orders of magnitude higher than the methanol deuteration measured in massive hot cores. Dust temperature maps obtained from Herschel and Planck observations show that massive hot cores are located in warmer molecular clouds than low-mass sources, with temperature differences of $\sim 10 \mathrm{~K}$. The comparison of our measured values with the predictions of the gas-grain astrochemical model GRAINOBLE shows that such a temperature difference is sufficient to explain the different deuteration observed in low- to high-mass sources. This suggests that the physical conditions of the molecular cloud at the origin of the protostars mostly govern the present-day observed deuteration of methanol and therefore of more complex organic molecules. Finally, the methanol deuteration measured towards young solar-type protostars on Solar System scales seems to be higher by a factor of $\sim 5$ than the upper limit in methanol deuteration estimated in comet Hale-Bopp. If this result is confirmed by subsequent observations of other comets, it would imply that an important reprocessing of the organic material likely occurred in the solar nebula during the formation of the Solar System.
\end{abstract}

Key words. astrochemistry - molecular processes - ISM: abundances - ISM: molecules - submillimeter: ISM - stars: formation

\section{Introduction}

Low-mass protostars are known to be chemically rich with the detection of several dozen molecules (van Dishoeck et al. 1995; Cazaux et al. 2003; Herbst \& van Dishoeck 2009; Caux et al. 2011; Caselli \& Ceccarelli 2012; Jørgensen et al. 2016). A process of deuterium enrichment is also observed for several neutral species, in particular for molecules thought to be mostly formed in cold interstellar ices and then released in the warm gas surrounding protostars (Ceccarelli et al. 2014). So far, methanol and formaldehyde are the two molecules showing the highest deuterium fractionations, up to $50 \%$, or 5 orders of magnitude higher than the cosmic D/H elemental abundance ratio of $1.6 \times 10^{-5}$ (Linsky 2003; Ceccarelli et al. 1998; Parise et al. 2006). Other species that are thought to be also mostly formed in interstellar ices, such as water, show lower deuteration (Coutens et al. 2012;

\footnotetext{
* The reduced datacubes are only available at the CDS via anonymous ftp to cdsarc.u-strasbg. fr (130.79.128.5) or via http:// cdsarc.u-strasbg.fr/viz-bin/cat/J/A+A/632/A19
}

Taquet et al. 2013a; Persson et al. 2014). These differences have been invoked to reflect different moments or different mechanisms of formation (Cazaux et al. 2011; Taquet et al. 2013a, 2014; Ceccarelli et al. 2014).

Gas-phase chemistry is known to be inefficient for the formation of gaseous methanol (Geppert et al. 2006; Garrod et al. 2006). Instead, methanol has been found to be efficiently formed at the surface of cold interstellar grains in dark cloud conditions through the hydrogenation of $\mathrm{CO}$ and $\mathrm{H}_{2} \mathrm{CO}$ (Watanabe \& Kouchi 2002; Rimola et al. 2014). During the collapse of the protostellar envelope, solid methanol is released into the gas phase when the temperatures exceed $\sim 100 \mathrm{~K}$. The deuteration of methanol is thought to remain constant after its evaporation in the warm-gas phase of the inner protostellar regions because gas-phase chemistry is likely too slow to significantly alter the ratios during the lifetime of the protostar (Charnley et al. 1997; Osamura et al. 2004). As a consequence, the deuteration observed around low-mass protostars would be a fossil of its formation in the precursor dense cloud where interstellar 
ices formed. Taquet et al. (2012a, 2013a) showed that like other species, methanol deuteration is highly sensitive to the temperature and the density during ice formation. A deuteration higher than $1 \%$ is favoured by a methanol formation in cold $(T=10 \mathrm{~K})$ and dense $\left(n_{\mathrm{H}} \geq 10^{5} \mathrm{~cm}^{-3}\right)$ molecular cloud conditions.

Most measurements of the methanol deuteration carried out so far have been performed using single-dish millimeter facilities with beams larger than $10^{\prime \prime}$ (or 2000 AU at a typical distance of $200 \mathrm{pc}$ ). These large beams encompass the different components of the protostellar system: the small hot core where all the icy content is thermally evaporated, but also the cold external envelope and molecular outflows driven by the central source(s). The advent of modern millimeter interferometers, such as the IRAM Plateau de Bure Interferometer (IRAM-PdBI), now called the NOrthern Extended Millimeter Array (NOEMA), and the Atacama Large Millimeter/submillimeter Array (ALMA) now allows astronomers to zoom towards the inner protostellar regions, in the so-called hot corinos, to measure the deuteration of the whole icy content released in the gas phase. New measurements towards low-mass protostars within different nearby molecular clouds suggest lower methanol deuterations than those originally measured a decade ago with single-dish telescopes. Bianchi et al. (2017) derived a $\left[\mathrm{CH}_{2} \mathrm{DOH}\right] /\left[\mathrm{CH}_{3} \mathrm{OH}\right]$ abundance ratio of $2 \%$ in $\mathrm{HH} 212$, a low-mass protostar located in the Orion molecular cloud, whilst Jørgensen et al. (2018) estimated a $\left[\mathrm{CH}_{2} \mathrm{DOH}\right] /\left[\mathrm{CH}_{3} \mathrm{OH}\right]$ ratio of $7 \%$ in Source B of IRAS 16293 2422, a low-mass protobinary system located in the Ophiuchus cloud. In addition to being lower than previous single-dish measurements of $20-60 \%$, they also differ within each other by a factor of $\sim 3$. This difference might be due to different properties of the precursor clouds. The Orion molecular cloud is the closest massive star-forming region and is known to be more active and slightly warmer than the more quiescent Ophiuchus cloud. However, this assumption remains to be confirmed for sources located in other clouds.

This article presents an analysis of different published observational datasets from the IRAM-PdBI towards NGC 1333-IRAS 2A and -IRAS 4A, two bright Class 0 low-mass protostars located in the nearby Perseus molecular cloud. We also re-analyse partially published data from the ALMA towards $\mathrm{HH} 212$ in order to measure the $\left[\mathrm{CH}_{2} \mathrm{DOH}\right] /\left[\mathrm{CH}_{3} \mathrm{OH}\right]$ and $\left[\mathrm{CH}_{3} \mathrm{OD}\right] /\left[\mathrm{CH}_{3} \mathrm{OH}\right]$ abundance ratios in their hot corinos. In this article, we aim to investigate whether the warm methanol deuteration observed in hot cores is regulated by the dust temperature of precursor dark clouds. To this aim, we compare our data with interferometric observations towards more massive protostellar systems and with the predictions of a modern astrochemical model.

\section{Observations}

\subsection{Observational details}

The two low-mass Class 0 protostars IRAS 2A and IRAS 4A located in the NGC 1333 cloud at $299 \pm 14$ pc (Zucker et al. 2018) were observed with the IRAM PdBI at 143, 165, and $225 \mathrm{GHz}$. Observations at 143 and $165 \mathrm{GHz}$ were carried out on 2010 July 20, July 21, August 1, August 3, November 24, and 2011 March 10 , in the $\mathrm{C}$ and $\mathrm{D}$ configurations of the array. Observations at $225 \mathrm{GHz}$ were performed on 2011 November 27 and 28, on 2012 March 12, 15, 21, and 27, and on 2012 April, 2 in the B and $\mathrm{C}$ configurations of the array. A more detailed description of the observational setups is provided in Taquet et al. (2015) and Persson et al. (2014) for configurations B and C, respectively. The amplitude calibration uncertainty is estimated to be $\sim 20 \%$. The WIDEX correlator has been used at three frequency settings, providing a bandwidth of $3.6 \mathrm{GHz}$ each with a spectral resolution of $1.95 \mathrm{MHz}\left(4,3.5\right.$, and $2.6 \mathrm{~km} \mathrm{~s}^{-1}$ at 145,165 , and $225 \mathrm{GHz}$, respectively). The data calibration and imaging were performed using the CLIC and MAPPING packages of the GILDAS software ${ }^{1}$. Continuum images were produced by averaging line-free channels in the WIDEX correlator before the Fourier transformation of the data. The line spectral cubes were then obtained by subtracting the continuum visibilities from the whole (line+continuum) datacube, followed by natural weighted cleaning of individual channels. For IRAS 4A, the baseline has also been flattened by importing the data cubes into CLASS and subtracting a polynomial function of low order to each individual spectrum. Positions are given with respect to the continuum peaks of IRAS 2A and IRAS 4A1 located at $\alpha(\mathrm{J} 2000)=03^{\mathrm{h}} 28^{\mathrm{m}}$ $55^{\mathrm{s}} .57, \delta(\mathrm{J} 2000)=+31^{\circ} 14^{\prime} 37^{\prime \prime} .22$ and $\alpha(\mathrm{J} 2000)=03^{\mathrm{h}} 29^{\mathrm{m}} 10^{\mathrm{s}} .52$, $\delta(\mathrm{J} 2000)=+31^{\circ} 13^{\prime} 31^{\prime \prime}$.06, respectively.

The HH212-MM1 (hereafter HH212) protostar located in the Orion B cloud at about 400 pc (Kounkel et al. 2017) was observed in Band 7 with ALMA using $3412 \mathrm{~m}$ antennas between June 15 and July 19, 2014, during the Cycle 1 phase and $4412 \mathrm{~m}$ antennas between October 6 and November 26, 2016, during the Cycle 4 phase. The maximum baselines for the Cycle 1 and 4 observations are $650 \mathrm{~m}$ and $3 \mathrm{~km}$, respectively. In Cycle 1, the spectral windows between 337.1-338.9 GHz and 348.4-350.7 $\mathrm{GHz}$ were observed using spectral channels of $977 \mathrm{kHz}$ ( or $0.87 \mathrm{~km} \mathrm{~s}^{-1}$ ), subsequently smoothed to $1.0 \mathrm{~km}$ $\mathrm{s}^{-1}$ to increase sensitivity. Calibration was carried out following standard procedures, using quasars J0607-0834, J0541-0541, J0423-013, and Ganymede. For the Cycle 4 data, a single spectral window between $334.1-336.0 \mathrm{GHz}$ with a $488 \mathrm{kHz}$ (or $0.42 \mathrm{~km} \mathrm{~s}^{-1}$ ) spectral resolution, successively smoothed to $1 \mathrm{~km} \mathrm{~s}^{-1}$, was used. Calibration was carried out using quasars J0510+1800, J0552+0313, J0541-0211, and J0552-3627. Spectral line imaging was achieved using $\mathrm{CASA}^{2}$, whilst data analysis was performed using the GILDAS package. Positions are given with respect to the MM1 protostar continuum peak located at $\alpha(\mathrm{J} 2000)=05^{\mathrm{h}} 43^{\mathrm{m}} 51^{\mathrm{s}} .41, \delta(\mathrm{J} 2000)=-01^{\circ} 02^{\prime} 53^{\prime \prime} .17$. In addition to ${ }^{13} \mathrm{CH}_{3} \mathrm{OH}$ and $\mathrm{CH}_{2} \mathrm{DOH}$ images that have been presented by Bianchi et al. (2017), we also report the imaging of the $\mathrm{CH}_{3} \mathrm{OD}\left(6_{2-}-6_{1+}\right)$ transition at $335.1 \mathrm{GHz}$ (see also Codella et al. 2019). The size of the synthesised beams and the rms noise are reported in Table 1.

\subsection{Spectroscopic parameters}

We here focus on the $\mathrm{CH}_{2} \mathrm{DOH}, \mathrm{CH}_{3} \mathrm{OD}$, and $\mathrm{CHD}_{2} \mathrm{OH}$ deuterated isotopologues. We used the $\mathrm{CH}_{2} \mathrm{DOH}$ JPL data entry from Pearson et al. (2012). The new band strengths published by Pearson et al. (2012) can differ by a factor of a 1.5-2.0 from the values from Parise et al. (2002) that are used in most previous studies before 2012. With a vibrational correction factor of 1.15 at $160 \mathrm{~K}$ and 1.46 at $300 \mathrm{~K}$ based on the torsional data of Lauvergnat et al. (2009), the partition function is 1.3-2.0 higher than the partition function estimated by Parise et al. (2002). We re-analysed the $\mathrm{CH}_{2} \mathrm{DOH}$ transitions observed towards the IRAS 16293-2422 low-mass protostellar system with the IRAM 30-m telescope by Parise et al. (2002) to investigate the effect of these new spectroscopic data on the estimation of the $\mathrm{CH}_{2} \mathrm{DOH}$ column densities. We derived a $\mathrm{CH}_{2} \mathrm{DOH}$ column density 2.2 lower than the one found by these authors. For $\mathrm{CH}_{3} \mathrm{OD}$, we prepared a catalogue entry based on the band

\footnotetext{
http://wWw . iram. fr/IRAMFR/GILDAS

2 http://casa.nrao.edu
} 
Table 1. Parameters of the observational data.

\begin{tabular}{|c|c|c|c|}
\hline \multirow[b]{2}{*}{ Frequency } & \multicolumn{3}{|c|}{ NGC 1333-IRAS 2A (PdBI) } \\
\hline & $145 \mathrm{GHz}$ & $165 \mathrm{GHz}$ & $225 \mathrm{GHz}$ \\
\hline Beam size $\left({ }^{\prime \prime}\right)$ & $2.1 \times 1.7$ & $2.3 \times 1.7$ & $1.2 \times 1.0$ \\
\hline Beam PA $\left(^{\circ}\right)$ & 25 & 110 & 22 \\
\hline \multirow[t]{2}{*}{ rms(spectrum) ${ }^{(a)}$} & 2.6 & 3.5 & 2.9 \\
\hline & \multicolumn{3}{|c|}{ NGC 1333-IRAS 4A (PdBI) } \\
\hline Frequency & $145 \mathrm{GHz}$ & $165 \mathrm{GHz}$ & $225 \mathrm{GHz}$ \\
\hline Beam size ("') & $2.2 \times 1.7$ & $2.4 \times 1.8$ & $1.1 \times 0.8$ \\
\hline Beam PA $\left({ }^{\circ}\right)$ & 25 & 114 & 14 \\
\hline \multirow[t]{3}{*}{$\operatorname{rms}($ spectrum $){ }^{(a)}$} & 3.3 & 4.0 & 4.8 \\
\hline & \multicolumn{3}{|c|}{ HH212 (ALMA) } \\
\hline & $335 \mathrm{GHz}$ & $338 \mathrm{GHz}$ & $349 \mathrm{GHz}$ \\
\hline Beam size ("') & $0.15 \times 0.12$ & $0.52 \times 0.34$ & $0.41 \times 0.33$ \\
\hline Beam PA $\left({ }^{\circ}\right)$ & -88 & -63 & -63 \\
\hline rms(spectrum) ${ }^{(b)}$ & 1 & $5-6$ & $5-6$ \\
\hline
\end{tabular}

Notes. ${ }^{(a)}$ Units of $\mathrm{mJy}$ beam ${ }^{-1}$ channel $^{-1}$ for a channel width of $1.95 \mathrm{MHz}{ }^{(b)}$ Units of mJy beam ${ }^{-1}$ channel $^{-1}$ for a channel width of $0.87 \mathrm{~km} \mathrm{~s}^{-1}(335 \mathrm{GHz})$ and $1.0 \mathrm{~km} \mathrm{~s}^{-1}$ (at 338 and $\left.349 \mathrm{GHz}\right)$.

strengths published by Anderson et al. (1988) with frequencies updated by Duan et al. (2003). A self-consistent calculation of the $\mathrm{CH}_{3} \mathrm{OD}$ partition function has not be performed so far because the $\mathrm{CH}_{3} \mathrm{OD}$ spectrum has only been partially studied in the laboratory. Instead, we used two estimates for the $\mathrm{CH}_{3} \mathrm{OD}$ partition function $Z$ to derive the $\mathrm{CH}_{3} \mathrm{OD}$ column densities. The first function follows the estimation by Parise (2004) within the rigid-rotor approximation, giving $Z=1.42 T^{3 / 2}$ (see also Ratajczak et al. 2011), as used in most previous studies. The second follows the estimation by Belloche et al. (2016), whose partition function values are scaled with those of the $\mathrm{CH}_{3}^{18} \mathrm{OH}$ partition function (see also Jørgensen et al. 2018). At $150 \mathrm{~K}$, the partition function estimated by Parise (2004) is four times lower than the one estimated by Jørgensen et al. (2018), resulting in large differences in the derived $\mathrm{CH}_{3} \mathrm{OD}$ column densities. For $\mathrm{CHD}_{2} \mathrm{OH}$, we used the spectroscopic data and the partition function published by Parise et al. (2002). The $\mathrm{CHD}_{2} \mathrm{OH}$ partition function is found to be similar to that of $\mathrm{CH}_{2} \mathrm{DOH}$ published by the JPL database, with differences lower than $20 \%$ between 10 and $300 \mathrm{~K}$.

We also targeted transitions from $\mathrm{CH}_{2} \mathrm{DCN}$. The $\mathrm{CH}_{2} \mathrm{DCN}$ spectroscopic data were taken from the CDMS catalogue, based on Nguyen et al. (2013).

\section{Results}

\subsection{Integrated maps}

For IRAS 2A and IRAS 4A, the integrated emission maps of all methanol isotopologue transitions were obtained by integrating the flux over $V_{\text {sys }} \pm \Delta V$, where $V_{\text {sys }}$ is the source systemic velocity and $\Delta V$ is the transition full width at half-maximum (FWHM) line width. In practice, we integrated the line emission over three channels because the spectral resolution of the WIDEX data is low. Figures 1 and 2 give an overview of the maps obtained for $\mathrm{CH}_{2} \mathrm{DOH}, \mathrm{CH}_{3} \mathrm{OD}$, and $\mathrm{CHD}_{2} \mathrm{OH}$ in the three frequency settings. For all isotopologues, the emission is limited to the inner regions around the protostars within the synthesised beams. Towards IRAS 4A, the molecular emission originates from the north-western source IRAS 4A2, although the continuum emission of the sourth-eastern source IRAS 4A1 is brighter. These maps are in good agreement with previous interferometric observations of these sources, which suggest that the emission of complex organic molecules originates from hot corinos whose sizes are smaller than 0.'5 (or $150 \mathrm{au}$; Jørgensen et al. 2005; Persson et al. 2012; Maury et al. 2014; Taquet et al. 2015; López-Sepulcre et al. 2017; De Simone et al. 2017).

For $\mathrm{HH} 212$, we produced the integrated emission maps of ${ }^{13} \mathrm{CH}_{3} \mathrm{OH}, \mathrm{CH}_{2} \mathrm{DOH}$, and $\mathrm{CH}_{3} \mathrm{OD}$ transitions that are not blended with other transitions by integrating the flux over the entire line width after an analysis of their spectra towards the continuum peak. The Cycle 1 emission is spatially unresolved with a a beam of $0.5 \times 0.3$, corresponding to a size of $225 \times 135 \mathrm{au}$. However, the analysis of the higher angular resolution of Cycle 4 dataset provides a beam-deconvolved FWHM size of 0 . $^{\prime} 18 \times 00^{\prime} 12(81 \times 54 \mathrm{au})$ for the ${ }^{13} \mathrm{CH}_{3} \mathrm{OH}$ emission and $0.20 \times 0$. ' $10(90 \times 45 \mathrm{au})$ for $\mathrm{CH}_{2} \mathrm{DOH}$. As shown in Fig. 3, the emission originates from the hot-corino region and is confined to a rotating structure that extends at \pm 45 au from the equatorial plane and is elongated along and rotates around the jet axis. The actual interpretation of this emission is still debated and has been attributed to disc wind or a disc atmosphere from accretion shocks (Bianchi et al. 2017; Lee et al. 2017).

We measured the flux of all methanol isotopologue transitions integrated over an elliptical mask with similar size and position angle to the synthesised beam and with a centre at the molecular emission peak following the method described in Taquet et al. (2015). Tables A.1-A.6 summarise the properties and the measured flux of the targeted transitions towards the two sources.

\subsection{Population diagrams}

We used the so-called population diagram (PD) method described in Goldsmith et al. (1999) and Taquet et al. (2015) to investigate the effect of optical depth on the column densities of each level for the different methanol isotopologues. We performed a reduced chi-square $\left(\chi_{\text {red }}^{2}\right)$ minimization by running a grid of models covering a large parameter space in rotational temperature $T_{\text {rot }}$ between 50 and $350 \mathrm{~K}$, and total column density in the source $N_{\text {tot }}$ between $10^{15}$ and $10^{20} \mathrm{~cm}^{-2}$. We assumed the source sizes $\theta_{\mathrm{s}}$ derived in Taquet et al. (2015) and Bianchi et al. (2017). A source size of 0.'36 and 0.'20 in IRAS 2A and IRAS 4A, respectively, was needed to reproduce the scatter of the population distribution of the low $\mathrm{CH}_{3} \mathrm{OH}$ upper energy levels (see Taquet et al. 2015), and a source size of 0.'19 was derived by Bianchi et al. (2017) from the analysis of the spatially resolved ${ }^{13} \mathrm{CH}_{3} \mathrm{OH}$ Cycle 4 emission map. At local thermal equilibrium (LTE), the column density of every upper state $N_{\text {up }}$ can be derived for each set of $N_{\text {tot }}, T_{\text {rot }}$, and source solid angle $\Omega_{\mathrm{s}}$. The best-fit model populations are plotted together with the observed populations of the levels in Figs. 4-6 and are marked by crosses. Table 2 summarises the parameters of the best-fit models and their associated uncertainties. The column densities of $\mathrm{CH}_{3} \mathrm{OH}$ and $\mathrm{CH}_{3} \mathrm{CN}$ have been derived in Taquet et al. (2015) by analysing the emission from $28 \mathrm{CH}_{3} \mathrm{OH}$ and $13{ }^{13} \mathrm{CH}_{3} \mathrm{OH}$ transitions assuming a ${ }^{12} \mathrm{C} /{ }^{13} \mathrm{C}$ elemental ratio of 70 and from six $\mathrm{CH}_{3} \mathrm{CN}$ transitions detected in the 145 and $165 \mathrm{GHz}$ frequency settings, respectively. Taquet et al. (2015) used the same PD method by considering the rotational temperature $T_{\text {rot }}$, the total column density in the source $N_{\text {tot }}$, and the source size $\theta_{\mathrm{S}}$ as free parameters. 

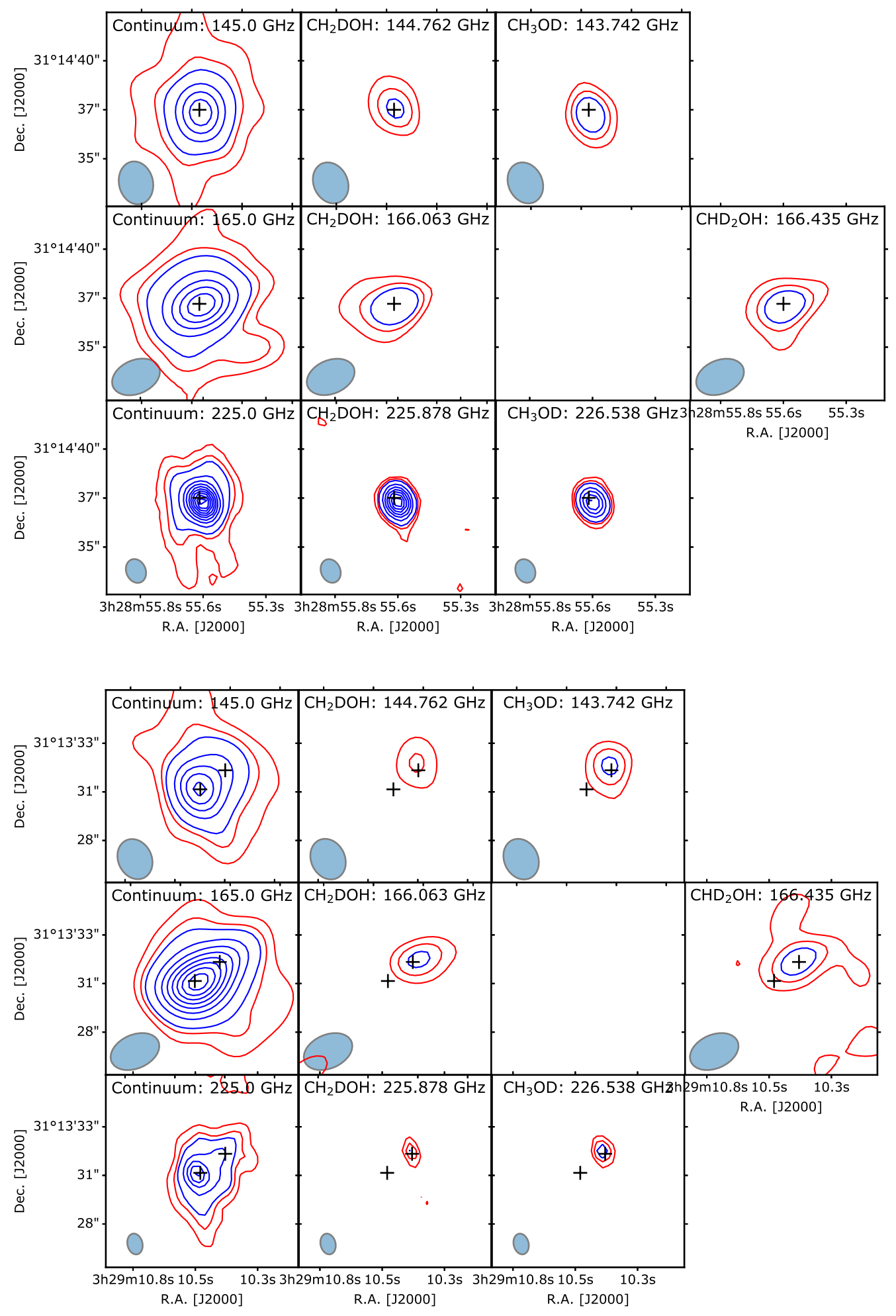

Fig. 1. Integrated continuum maps, $\mathrm{CH}_{2} \mathrm{DOH}, \mathrm{CH}_{3} \mathrm{OD}$, and $\mathrm{CHD}_{2} \mathrm{OH}$ emission observed towards IRAS 2A for the three PdBI frequency settings (top: 145 $\mathrm{GHz}$, middle: $165 \mathrm{GHz}$, and bottom: $225 \mathrm{GHz}$ ). Red contours show the $3 \sigma$ and $6 \sigma$ levels, whilst blue contours are in steps of $9 \sigma$. The synthesised beams are shown in the bottom left corner of each panel. The black cross depicts the position of the protostar.

Fig. 2. Integrated continuum maps, $\mathrm{CH}_{2} \mathrm{DOH}, \mathrm{CH}_{3} \mathrm{OD}$, and $\mathrm{CHD}_{2} \mathrm{OH}$ emission observed towards IRAS 4A for the three PdBI frequency settings (top: $145 \mathrm{GHz}$, middle: $165 \mathrm{GHz}$, and bottom: $225 \mathrm{GHz}$ ). Red contours show the $3 \sigma$ and $6 \sigma$ levels, whilst blue contours are in steps of $9 \sigma$. The synthesised beams are shown in the bottom left corner of each panel. The black crosses depict the position of the protostars.

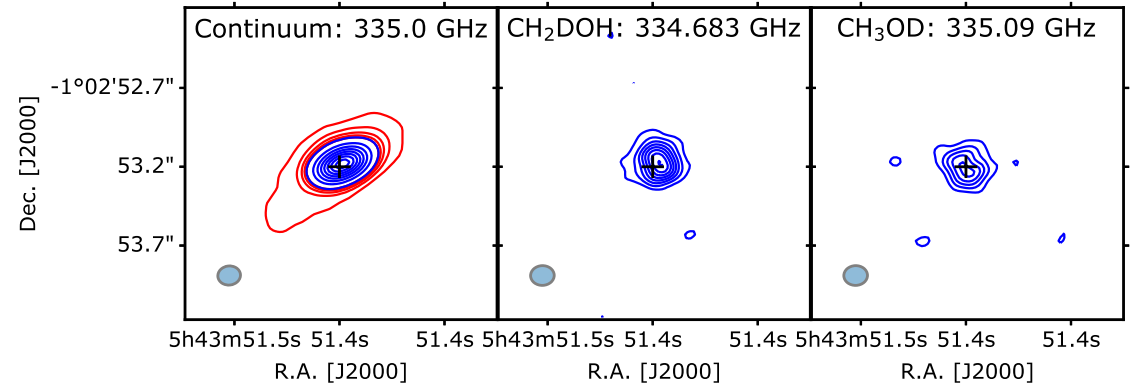

Fig. 3. Integrated continuum maps, $\mathrm{CH}_{2} \mathrm{DOH}$, and $\mathrm{CH}_{3} \mathrm{OD}$ emission observed towards $\mathrm{HH} 212$ for the ALMA frequency setting at $335 \mathrm{GHz}$. For the continuum map, red contours show the 5 first $20 \sigma$ levels and blue contours are in steps of $100 \sigma$. For the molecular maps, blue contours are in steps of $3 \sigma$. The synthesised beams are shown in the bottom left corner of each panel. 
Table 2. Results from the PD analysis for the methanol and methyl cyanide deuterated isotopologues.

\begin{tabular}{|c|c|c|c|c|c|}
\hline Molecule & $\begin{array}{c}N_{\mathrm{hc}} \\
\left(\mathrm{cm}^{-2}\right)\end{array}$ & $\begin{array}{l}T_{\text {rot }} \\
(\mathrm{K})\end{array}$ & $\begin{array}{l}\text { Source size } \\
\left({ }^{\prime \prime}\right)\end{array}$ & $\begin{array}{c}N(\mathrm{XD}) / N(\mathrm{XH})^{(a)} \\
(\%)\end{array}$ & $\begin{array}{c}N(\mathrm{XD}) / N(\mathrm{XH})^{(b)} \\
(\text { Single-dish, \%) }\end{array}$ \\
\hline \multicolumn{6}{|c|}{ IRAS $2 A$} \\
\hline $\mathrm{CH}_{3} \mathrm{OH}$ & $\left(5.0_{-1.8}^{+2.9}\right)(+18)$ & $140_{-20}^{+20}$ & $0.36_{-0.04}^{+0.04(c)}$ & - & - \\
\hline${ }^{13} \mathrm{CH}_{3} \mathrm{OH}$ & $\left(7.1_{-2.6}^{+4.2}\right)(+16)$ & $140_{-20}^{+20}$ & $0.36_{-0.04}^{+0.04}(c)$ & - & - \\
\hline $\mathrm{CH}_{2} \mathrm{DOH}$ & $\left(2.9_{-0.6}^{+1.2}\right)(+17)$ & $166_{-48}^{+58}$ & $0.36^{(d)}$ & $5.8_{-1.5}^{+1.3}$ & $62_{-33}^{+71}$ \\
\hline $\mathrm{CH}_{3} \mathrm{OD}\left(\mathrm{P} 04^{(e)}\right)$ & $\left(7.9_{-1.6}^{+2.1}\right)(+16)$ & $166^{(f)}$ & $0.36^{(d)}$ & $1.6_{-0.8}^{+0.6}$ & $\leq 8$ \\
\hline $\mathrm{CH}_{3} \mathrm{OD}\left(\mathrm{J}_{18}{ }^{(e)}\right)$ & $\left(3.6_{-0.4}^{+0.9}\right)(+17)$ & $166^{(f)}$ & $0.36^{(d)}$ & $7.1_{-1.6}^{+1.2}$ & $\leq 8$ \\
\hline $\mathrm{CHD}_{2} \mathrm{OH}$ & $\left(2.2_{-0.5}^{+0.6}\right)(+17)$ & $164_{-32}^{+44}$ & $0.36^{(d)}$ & $4.4_{-1.3}^{+0.9}$ & $25_{-14}^{+29}$ \\
\hline $\mathrm{CH}_{3} \mathrm{CN}$ & $\left(2.0_{-0.4}^{+1.2}\right)(+16)$ & $130_{-40}^{+230}$ & $0.36^{(c)}$ & - & - \\
\hline $\mathrm{CH}_{2} \mathrm{DCN}$ & $\left(7.2_{-0.9}^{+1.5}\right)(+14)$ & $130^{(g)}$ & $0.36^{(g)}$ & $3.6_{-1.2}^{+0.5}$ & - \\
\hline \multicolumn{6}{|c|}{ IRAS 4A } \\
\hline $\mathrm{CH}_{3} \mathrm{OH}$ & $\left(1.6_{-0.8}^{+0.6}\right)(+19)$ & $140_{-30}^{+30}$ & $0.20_{-0.04}^{+0.08(c)}$ & - & - \\
\hline${ }^{13} \mathrm{CH}_{3} \mathrm{OH}$ & $\left(2.3_{-1.1}^{+1.3}\right)(+17)$ & $140_{-30}^{+30}$ & $0.20_{-0.04}^{+0.08(c)}$ & - & - \\
\hline $\mathrm{CH}_{2} \mathrm{DOH}$ & $\left(5.9_{-1.8}^{+2.2}\right)(+17)$ & $152_{-78}^{+62}$ & $0.20^{(d)}$ & $3.7_{-0.9}^{+1.2}$ & $65_{-21}^{+30}$ \\
\hline $\mathrm{CH}_{3} \mathrm{OD}\left(\mathrm{P} 04^{(e)}\right)$ & $\left(1.1_{-0.2}^{+0.5}\right)(+17)$ & $152^{(f)}$ & $0.20^{(d)}$ & $0.7_{-0.4}^{+0.5}$ & $4.7_{-2.1}^{+2.9}$ \\
\hline $\mathrm{CH}_{3} \mathrm{OD}\left(\mathrm{J}_{18}{ }^{(e)}\right)$ & $\left(5.0_{-1.0}^{+1.3}\right)(+17)$ & $152^{(f)}$ & $0.20^{(e)}$ & $3.1_{-0.8}^{+1.0}$ & $4.7_{-2.1}^{+2.9}$ \\
\hline $\mathrm{CHD}_{2} \mathrm{OH}$ & $\left(3.3_{-0.7}^{+0.9}\right)(+17)$ & $138_{-38}^{+34}$ & $0.20^{(d)}$ & $2.1_{-0.6}^{+0.8}$ & $\begin{array}{r}17_{-6}^{+9} \\
\end{array}$ \\
\hline $\mathrm{CH}_{3} \mathrm{CN}$ & $\left(6.3_{-1.3}^{+3.6}\right)(+16)$ & $200_{-40}^{+110}$ & $0.20^{(c)}$ & - & - \\
\hline $\mathrm{CH}_{2} \mathrm{DCN}$ & $\left(1.7_{-0.7}^{+0.7}\right)(+15)$ & $200^{(g)}$ & $0.20^{(g)}$ & $2.7_{-1.1}^{+0.7}$ & - \\
\hline \multicolumn{6}{|c|}{ HH212 } \\
\hline${ }^{13} \mathrm{CH}_{3} \mathrm{OH}$ & $\left(3.2_{-1.4}^{+1.4}\right)(+16)$ & $155_{-65}^{+69}$ & $0.19^{(h)}$ & - & - \\
\hline $\mathrm{CH}_{2} \mathrm{DOH}$ & $\left(6.4_{-1.3}^{+1.3}\right)(+16)$ & $155^{(i)}$ & $0.19^{(h)}$ & $2.9_{-0.8}^{+0.8}$ & - \\
\hline $\mathrm{CH}_{3} \mathrm{OD}\left(\mathrm{P} 04^{(e)}\right)$ & $\left(9.9_{-1.2}^{+1.2}\right)(+15)$ & $155^{(i)}$ & $0.19^{(h)}$ & $0.4_{-0.3}^{+0.3}$ & - \\
\hline $\mathrm{CH}_{3} \mathrm{OD}\left(\mathrm{J}_{18}{ }^{(e)}\right)$ & $\left(4.4_{-0.5}^{+0.5}\right)(+16)$ & $155^{(i)}$ & $0.19^{(h)}$ & $2.0_{-0.6}^{+0.6}$ & - \\
\hline
\end{tabular}

Notes. ${ }^{(a)}$ Column density ratio between a deuterated species and its main isotopologue. ${ }^{(b)}$ From Parise et al. $(2006) .{ }^{(c)}$ From Taquet et al. $(2015)$. ${ }^{(d)}$ The source size was assumed to be equal to that of $\mathrm{CH}_{3} \mathrm{OH} .{ }^{(e)} \mathrm{P} 04$ refers to the partition function computed by Parise (2004) within the rigidrotor approximation, whilst $\mathrm{J} 18$ refers to the partition function used in Jørgensen et al. (2018) obtained from a scaling of that of $\mathrm{CH}_{3}^{18} \mathrm{OH}$. ${ }^{(f)} \mathrm{The}$ rotational temperature was assumed to be equal to that of $\mathrm{CH}_{2} \mathrm{DOH}$. ${ }^{(g)}$ The rotational temperature was assumed to be equal to that of $\mathrm{CH}_{3} \mathrm{CN}$. ${ }^{(h)}$ From Bianchi et al. (2017). ${ }^{(i)}$ The rotational temperature was assumed to be equal to that of ${ }^{13} \mathrm{CH}_{3} \mathrm{OH}$.

For IRAS $2 \mathrm{~A}$ and IRAS 4A, we started the analysis with $\mathrm{CH}_{2} \mathrm{DOH}$ because this is the isotopologue with the highest number of detected transitions and with the widest excitation range, with 25 detected transitions of upper level energies $E_{\text {up }}$ between 33 and $364 \mathrm{~K}$. The population distributions can be reproduced with rotational temperatures of $166_{-48}^{+58}$ and $152_{-78}^{+62} \mathrm{~K}$ in IRAS 2A and IRAS 4A, respectively. These temperatures are slightly higher, but within the uncertainties, than the temperature of $140 \mathrm{~K}$ needed to reproduce the $\mathrm{CH}_{3} \mathrm{OH}$ and ${ }^{13} \mathrm{CH}_{3} \mathrm{OH}$ emissions (Taquet et al. 2015). Transitions with upper level energies lower than $100 \mathrm{~K}$ are optically thick with opacities of $0.2-0.4$ towards IRAS 2A and 0.5-1.0 towards IRAS 4A, depending on their properties. For $\mathrm{CH}_{3} \mathrm{OD}$, we assumed the same rotational temperature as for $\mathrm{CH}_{2} \mathrm{DOH}$ because of the lower range of excitation ( $E_{\text {up }}$ from 33 to $104 \mathrm{~K}$ ) and the high scatter and uncertainties in the population distributions for both sources. The strong scatter of the $\mathrm{CH}_{3} \mathrm{OD}$ population is not entirely reproduced, possibly because of non-LTE effets due to high critical densities. $\mathrm{CH}_{3} \mathrm{OD}$ transitions are also optically thick with opacities ranging from 0.3 to 1.3 towards the two sources. The $\mathrm{CHD}_{2} \mathrm{OH}$ population distribution shows much less scatter that $\mathrm{CH}_{3} \mathrm{OD}$. The comparison of the column densities derived in this work with those found for $\mathrm{CH}_{3} \mathrm{OH}$ in Taquet et al. (2015) allows us to derive the methanol deuterations in the two sources. We find a $\left[\mathrm{CH}_{2} \mathrm{DOH}\right] /\left[\mathrm{CH}_{3} \mathrm{OH}\right]$ abundance ratio of $5.8 \pm 0.8$ and $3.2 \pm 0.7 \%$ towards IRAS $2 \mathrm{~A}$ and IRAS 4A, respectively. Using the $\mathrm{CH}_{3} \mathrm{OD}$ partition function estimated by Parise (2004), we find $\left[\mathrm{CH}_{2} \mathrm{DOH}\right] /\left[\mathrm{CH}_{3} \mathrm{OD}\right]$ abundance ratios of $3.6 \pm 1.6$ and $5.3 \pm 3.4$ in IRAS $2 \mathrm{~A}$ and IRAS $4 \mathrm{~A}$, respectively. However, as expected, we find lower $\left[\mathrm{CH}_{2} \mathrm{DOH}\right] /\left[\mathrm{CH}_{3} \mathrm{OD}\right]$ abundance ratios of $0.8 \pm 0.3$ and $1.2 \pm 0.5$ in IRAS $2 \mathrm{~A}$ and IRAS 4A, respectively, when the partition function from Jørgensen et al. (2018) is used. As discussed in Sect. 4.3, a $\left[\mathrm{CH}_{2} \mathrm{DOH}\right] /\left[\mathrm{CH}_{3} \mathrm{OH}\right]$ abundance ratio of $\sim 3$ is consistent with the statistical value, whereas a ratio of $\sim 1$ remains puzzling. In addition to methanol, we also detect three transitions from the deuterated methyl cyanide 


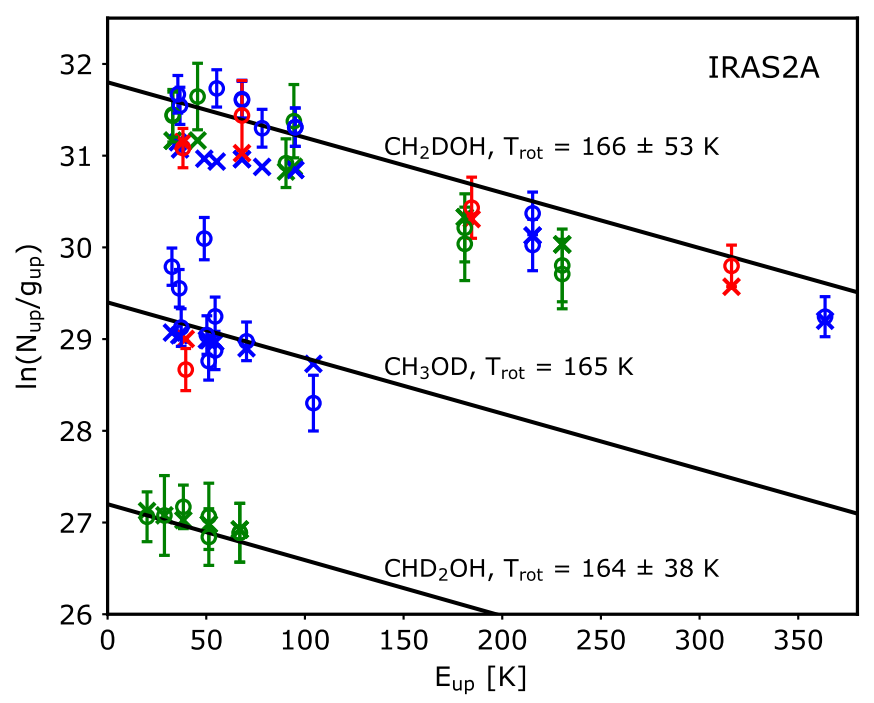

Fig. 4. Rotational and population diagrams of deuterated methanol isotopologues $\left(\mathrm{CH}_{2} \mathrm{DOH}, \mathrm{CH}_{3} \mathrm{OD}\right.$, and $\left.\mathrm{CHD}_{2} \mathrm{OH}\right)$ derived towards NGC 1333-IRAS 2A for source sizes derived from the PD analysis of the methanol population distribution ( 0 '.36; see Taquet et al. 2015). Red, green, and blue symbols represent transitions at 145, 165, and $225 \mathrm{GHz}$, respectively. The $\mathrm{CH}_{3} \mathrm{OD}$ and $\mathrm{CHD}_{2} \mathrm{OH}$ levels have been artificially shifted by -1.5 and -4 for clarity. Observational data are depicted by diamonds. Crosses show the best fit of the PD to the data. Blue and green symbols represent transitions at 2 and $1.3 \mathrm{~mm}$, respectively. Error bars are derived assuming a calibration uncertainty of $20 \%$ in addition to the statistical error. Black straight lines represent the best fit of the rotational diagram analysis to the data, assuming optically thin emission.

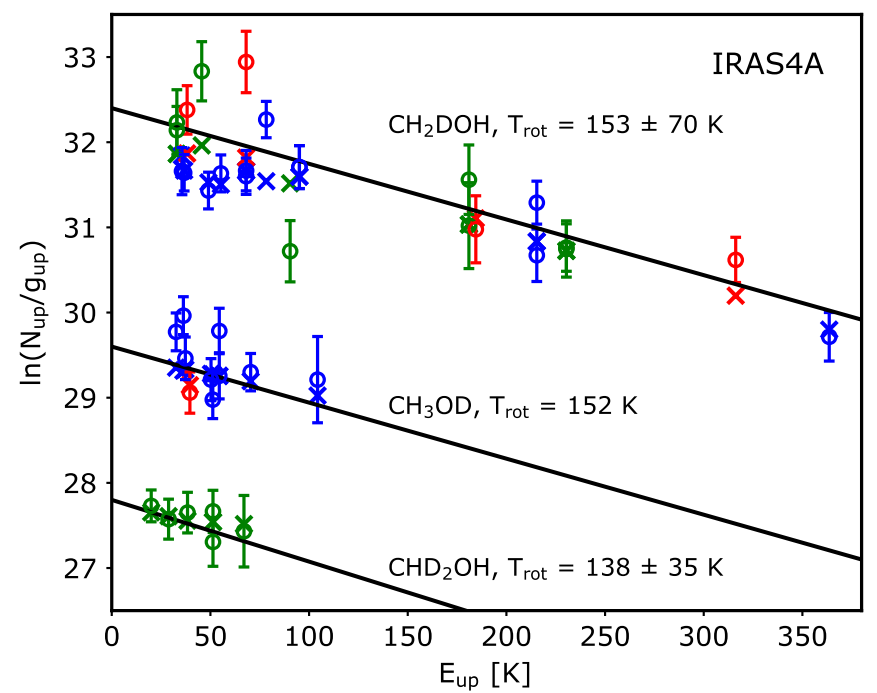

Fig. 5. Rotational and population diagrams of deuterated methanol isotopologues $\left(\mathrm{CH}_{2} \mathrm{DOH}, \mathrm{CH}_{3} \mathrm{OD}\right.$, and $\left.\mathrm{CHD}_{2} \mathrm{OH}\right)$ derived towards NGC 1333-IRAS 2A for source sizes derived from the PD analysis of the methanol population distribution ( 0 '20; see Taquet et al. 2015). Red, green, and blue symbols represent transitions at 145,165 , and $225 \mathrm{GHz}$, respectively. The $\mathrm{CH}_{3} \mathrm{OD}$ and $\mathrm{CHD}_{2} \mathrm{OH}$ levels have been artificially shifted by -1.5 and -4 for clarity. Observational data are depicted by diamonds. Crosses show the best fit of the PD to the data. Blue and green symbols represent transitions at 2 and $1.3 \mathrm{~mm}$, respectively. Error bars are derived assuming a calibration uncertainty of $20 \%$ in addition to the statistical error. Black straight lines represent the best fit of the rotational diagram analysis to the data, assuming optically thin emission.

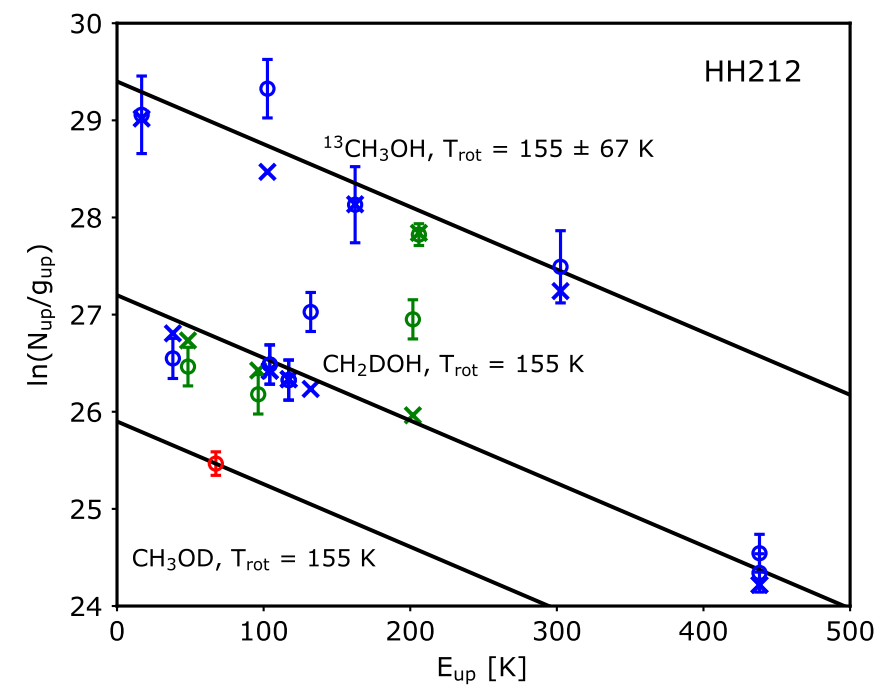

Fig. 6. Rotational and population diagrams of methanol isotopologues $\left({ }^{13} \mathrm{CH}_{3} \mathrm{OH}, \mathrm{CH}_{2} \mathrm{DOH}\right.$, and $\left.\mathrm{CH}_{3} \mathrm{OD}\right)$ derived towards $\mathrm{HH} 212$ for the source sizes derived from the ALMA Cycle 4 data $(0$.' 19 ; see Bianchi et al. 2017). The $\mathrm{CH}_{2} \mathrm{DOH}$ and $\mathrm{CH}_{3} \mathrm{OD}$ levels have been artificially shifted by -3 for clarity. Red, green, and blue symbols represent transitions at 335,338 , and $349 \mathrm{GHz}$, respectively. Observational data are depicted by diamonds. Crosses show the best fit of the PD to the data. Black straight lines represent the best fit of the rotational diagram analysis to the data, assuming optically thin emission.

isotopologue $\mathrm{CH}_{2} \mathrm{DCN}$ at $225 \mathrm{GHz}$. We obtain the $\mathrm{CH}_{2} \mathrm{DCN}$ column densities by assuming that the $\mathrm{CH}_{2} \mathrm{DCN}$ rotational temperature is equal to $200 \mathrm{~K}$, the rotational temperature of $\mathrm{CH}_{3} \mathrm{CN}$ measured in Taquet et al. (2015). Comparing the $\mathrm{CH}_{2}$ DCN column densities derived in this work with those of $\mathrm{CH}_{3} \mathrm{CN}$ from Taquet et al. (2015) allows us to obtain $\left[\mathrm{CH}_{2} \mathrm{DCN}\right] /\left[\mathrm{CH}_{3} \mathrm{CN}\right]$ ratios of $3.6 \pm 0.8$ and $2.7 \pm 0.9 \%$ in IRAS $2 \mathrm{~A}$ and IRAS $4 \mathrm{~A}$, respectively.

For $\mathrm{HH} 212$, we re-derived the column densities of ${ }^{13} \mathrm{CH}_{3} \mathrm{OH}$ and $\mathrm{CH}_{2} \mathrm{DOH}$ that have been estimated by Bianchi et al. (2017). Our method differs slightly from that of Bianchi et al. (2017) because the PD diagram is built upon the flux extracted from an elliptical mask instead of the continuum peak position in order to use consistent methods for the different sources. Assuming a source size of 0 .' 19 following Bianchi et al. (2017), we started with ${ }^{13} \mathrm{CH}_{3} \mathrm{OH}$ and then used the derived rotational temperature to estimate the $\mathrm{CH}_{2} \mathrm{DOH}$ and $\mathrm{CH}_{3} \mathrm{OD}$ column densities. We obtain a $\left[\mathrm{CH}_{2} \mathrm{DOH}\right] /\left[\mathrm{CH}_{3} \mathrm{OH}\right]$ abundance ratio of $2.9 \pm 0.8 \%$. The $\left[\mathrm{CH}_{2} \mathrm{DOH}\right] /\left[\mathrm{CH}_{3} \mathrm{OD}\right]$ abundance ratio is found to be $7.2 \pm 5.3$ and $1.5 \pm 0.6$ with the $\mathrm{CH}_{3} \mathrm{OD}$ partition function estimated by Parise (2004) and Jørgensen et al. (2018), respectively.

\section{Discussion}

\subsection{Comparison with previous observations}

Deuterated methanol has previously been detected with the IRAM 30-m single-dish telescope towards IRAS 2A and IRAS 4A. Parise et al. (2006) derived $\left[\mathrm{CH}_{2} \mathrm{DOH}\right] /\left[\mathrm{CH}_{3} \mathrm{OH}\right]$ abundance ratios of 62 and $65 \%$ towards IRAS $2 A$ and IRAS 4A, respectively, which are 10 and 17 times higher than the deuteration values found in this study. The $\left[\mathrm{CH}_{3} \mathrm{OD}\right] /\left[\mathrm{CH}_{3} \mathrm{OH}\right]$ and $\left[\mathrm{CH}_{2} \mathrm{DOH}\right] /\left[\mathrm{CH}_{3} \mathrm{OD}\right]$ abundance ratios derived in this work highly depend on the partition function that is used (see 
Sect. 2.2). With the same partition function as Parise (2004), the $\left[\mathrm{CH}_{3} \mathrm{OD}\right] /\left[\mathrm{CH}_{3} \mathrm{OH}\right]$ ratio of $4.7 \%$ found by Parise et al. (2006) towards IRAS $4 \mathrm{~A}$ is 6.7 times higher than our derived value, whilst the $\left[\mathrm{CH}_{2} \mathrm{DOH}\right] /\left[\mathrm{CH}_{3} \mathrm{OD}\right]$ is found to decrease by a factor of 4 between Parise et al. (2006) and this work. For doubly deuterated methanol, the $\left[\mathrm{CHD}_{2} \mathrm{OH}\right] /\left[\mathrm{CH}_{3} \mathrm{OH}\right]$ ratios derived in this work are also much lower than the abundances derived by Parise et al. (2006): by a factor of 5.6 and 8.1 for IRAS $2 A$ and IRAS 4A, respectively.

The large differences found between Parise et al. (2006) and the present work can be explained by several factors. First, the new $\mathrm{CH}_{2} \mathrm{DOH}$ spectroscopic data by Pearson et al. (2012) used in this work decrease by a factor of two the derived $\mathrm{CH}_{2} \mathrm{DOH}$ column density following a re-analysis of the single-dish data towards IRAS 2A by Parise et al. (2006) using the most recent spectroscopy data entry. This also naturally explains the increase of the $\left[\mathrm{CHD}_{2} \mathrm{OH}\right] /\left[\mathrm{CH}_{2} \mathrm{DOH}\right]$ ratio in this work by a factor of about two, from 41 to $75 \%$ in IRAS $2 \mathrm{~A}$ and from 26 to $56 \%$ in IRAS 4 A. Second, the methanol deuteration derived from single-dish observations by Parise et al. (2006) has been estimated from the detection of various transitions from the main $\mathrm{CH}_{3} \mathrm{OH}$ isotopologue. However, the targeted transitions have low upper level energies $\left(E_{\text {up }}<150 \mathrm{~K}\right)$ and are optically thick in the inner protostellar regions surrounding these two low-mass protostars (see Taquet et al. 2015). Therefore, the rotational diagram analysis, assuming optically thin emission, carried out in Parise et al. (2006) could have likely led to an underestimation of the $\mathrm{CH}_{3} \mathrm{OH}$ column density. Finally, the IRAM 30-m telescope has a large beam of 9-30" depending on the frequency. As suggested by the low rotation temperatures of 55 and $27 \mathrm{~K}$ found by Parise et al. (2006) for $\mathrm{CH}_{2} \mathrm{DOH}$ towards IRAS 2A and IRAS 4A, respectively, the methanol emission would mostly come from the large external envelope and/or from the molecular outflows driven by the targeted sources where methanol deuteration is higher. Methanol is mostly produced through surface chemistry through $\mathrm{CO}$ hydrogenation as gas phase chemistry is known to be inefficient (Watanabe \& Kouchi 2002; Geppert et al. 2006). Non-thermal evaporation processes could release a fraction of solid methanol formed in ices into the gas phase. As shown by Taquet et al. (2014), the deuteration of methanol and other molecules mostly produced in ices like water is higher in external protostellar envelopes than in the inner hot cores because the cold gaseous deuteration reflects the deuteration at the highly deuterated ice surface.

Deuterated methanol has not previously been targeted with single-dish facilities towards $\mathrm{HH} 212$. Our PD analysis gives a $\left[\mathrm{CH}_{2} \mathrm{DOH}\right] /\left[\mathrm{CH}_{3} \mathrm{OH}\right]$ abundance ratio of $2.9 \pm 0.8 \%$, which agrees well with the value of $2.4 \pm 0.4 \%$ found by Bianchi et al. (2017), who used a slightly different method.

\subsection{Deuteration from low- to high-mass protostars}

Figure 7 and Table 3 compare the deuteration of $\mathrm{CH}_{2} \mathrm{DOH}$, $\mathrm{CH}_{3} \mathrm{OD}$, and $\mathrm{CH}_{2} \mathrm{DCN}$, including their statistical (i.e. taking into account the statistical ratios for the functional groups with multiple $\mathrm{H}$-atoms) correction, between four low-mass, one intermediate-mass, and three massive hot cores obtained only through interferometric observations of methanol isotopologues We also show the water deuteration for comparison when available. Because the main $\mathrm{CH}_{3} \mathrm{OH}$ isotopologue is known to be optically thick in hot cores, we only chose observations whose deuteration values were derived from ${ }^{13} \mathrm{CH}_{3} \mathrm{OH}$ or $\mathrm{CH}_{3}^{18} \mathrm{OH}$ column densities assuming ${ }^{12} \mathrm{C} /{ }^{13} \mathrm{C}$ and ${ }^{16} \mathrm{O} /{ }^{18} \mathrm{O}$ elemental ratios of 70 and 560, respectively (Wilson \& Rood 1994). IRAS 16293 2422, a low-mass protobinary system located in the Ophiuchus molecular cloud, has been observed with ALMA by Jørgensen et al. (2018) in the context of the PILS survey that covered the whole ALMA band 7 between 329.15 and $362.90 \mathrm{GHz}$ (Jørgensen et al. 2016). NGC 7129 FIRS 2, an intermediatemass protostar, has been observed with the IRAM-PdBI at 220 $\mathrm{GHz}$ by Fuente et al. (2014). The methanol deuterium fractionation towards the Orion KL massive star-forming region has been estimated by Peng et al. (2012) through interferometric observations using the IRAM-PdBI at 103 and $225 \mathrm{GHz}$. The Sgr B2(N2) massive hot core has been observed by Belloche et al. (2016) in the context of the EMoCA ALMA survey of the whole $3 \mathrm{~mm}$ band. The NGC 6334 massive protocluster has recently been observed by Bøgelund et al. (2018) through ALMA observations at $300 \mathrm{GHz}$, and we selected the deuteration derived using the $\mathrm{CH}_{3}^{18} \mathrm{OH}$ column density. The error bars of the $\mathrm{CH}_{3} \mathrm{OD}$ deuterations derived in this work include the different column density values derived with the two partition functions used for $\mathrm{CH}_{3} \mathrm{OD}$.

The dust temperature in the dark clouds surrounding protostars is mostly governed by the external interstellar radiation field with local variations due to bright stars and/or dense cores. All the hot cores considered in Fig. 7 and Table 3 are likely relatively young, that is, have a lifetime of $10^{5} \mathrm{yr}$ at most, the dust temperatures of their precursor dark clouds are therefore likely similar to the current observed values. The deuterium fractionation is plotted against the current dust temperature of their surrounding cloud as measured by the Planck observatory for NGC 7129 FIRS2 and by the PACS and SPIRE instruments on board the Herschel Space Observatory for other sources. The Ophiuchus, Perseus, and Orion molecular clouds have been observed with the Gould Belt Survey key program (André et al. 2010), the Sgr B2 region has been observed by Etxaluze et al. (2013) as part of the Hi-GAL key program (Molinari et al. 2010), whilst the NGC 6334 massive complex has been observed with the HOBYS key program (Motte et al. 2010; Russeil et al. 2013; Tige et al. 2017). To estimate the dust temperature, we extracted 10 arcmin maps surrounding the selected sources. We then generated temperature histograms of all pixels showing visual extinctions $A_{\mathrm{V}}$ higher than 3 mag when the $\mathrm{H}_{2}$ column density maps are available (for the Ophiuchus, Perseus, and NGC 6334 clouds) or showing $353 \mathrm{GHz}$ opacities higher than ten times the rms noise (for the Orion and NGC 7129 clouds). To derive a temperature representative of the entire region, we filtered out the hot regions that are locally heated by stars or cold regions of dense cores by fitting the temperature histogram around the main peak with a Gaussian function. Figure B.1 gives an example for the NGC1333 region containing IRAS $2 \mathrm{~A}$ and IRAS 4A.

It is found that the statistical deuterium fractionations of $\mathrm{CH}_{2} \mathrm{DOH}$ and $\mathrm{CH}_{3} \mathrm{OD}$ tend to decrease from a few percent to less than $\sim 0.1 \%$ with the dust temperature between $\sim 13$ and $\sim 24 \mathrm{~K}$. With the same partition function as estimated by Belloche et al. (2016) and Jørgensen et al. (2018), the $\left[\mathrm{CH}_{2} \mathrm{DOH}\right] /\left[\mathrm{CH}_{3} \mathrm{OD}\right]$ abundance ratio seems to show a large dispersion because it typically varies from 1-4 in low-mass protostars to $0.1-2$ in high-mass hot cores $(0.8 \pm 0.3$ in IRAS $2 \mathrm{~A}$, $1.2 \pm 0.5$ in IRAS 4 A, $3.9 \pm 1.1$ in IRAS $16293,1.5 \pm 0.6$ in $\mathrm{HH} 212,1.7$ in Sgr B2, $0.73 \pm 0.27$ in Orion KL, and $0.13 \pm 0.03$ in NGC6334-IMM1).

It has been shown that warm hot-core gas-phase chemistry is likely too slow to significantly alter the deuteration after the evaporation of ices in hot cores (Charnley et al. 1997; Osamura et al. 2004). Thus, the methanol deuterium 

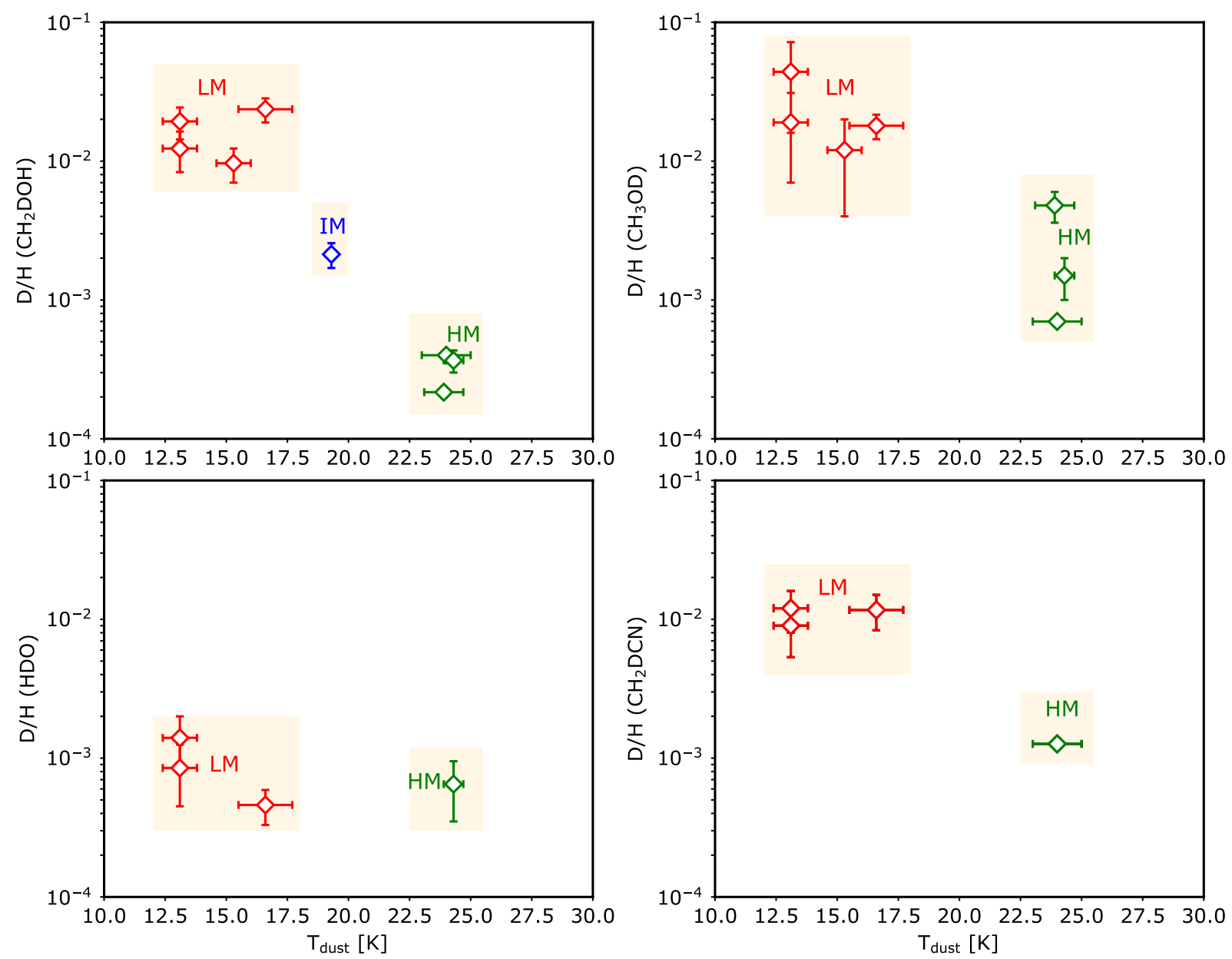

Fig. 7. Statistical (i.e. taking into account the statistical ratios for the functional groups with multiple $\mathrm{H}$-atoms) $\mathrm{CH}_{2} \mathrm{DOH}\left(\right.$ top left), $\mathrm{CH}_{3} \mathrm{OD}$ (top right), $\mathrm{HDO}$ (bottom left), and $\mathrm{CH}_{2} \mathrm{DCN}$ (bottom right) deuterium fractionations observed towards the hot core of a sample of low-mass (red), intermediate-mass (blue), and high-mass (green) protostars with sub-millimeter interferometers as function of the dust temperature of the surrounding cloud measured with the Herschel or Planck telescopes (see values in Table 3).

Table 3. Surrounding cloud dust temperatures and statistical deuterium fractionations observed towards protostellar hot cores.

\begin{tabular}{lccccccc}
\hline \hline Source & $\begin{array}{c}T_{\text {dust }} \\
(\mathrm{K})\end{array}$ & $\begin{array}{c}\mathrm{D} / \mathrm{H}\left(\mathrm{CH}_{2} \mathrm{DOH}\right) \\
(\%)\end{array}$ & $\begin{array}{c}\mathrm{D} / \mathrm{H}\left(\mathrm{CH}_{3} \mathrm{OD}\right) \\
(\%)\end{array}$ & $\begin{array}{c}{\left[\mathrm{CH}_{2} \mathrm{DOH}\right] /} \\
{\left[\mathrm{CH}_{3} \mathrm{OD}\right]}\end{array}$ & $\begin{array}{c}\mathrm{D} / \mathrm{H}\left(\mathrm{CH}_{2} \mathrm{DCN}\right) \\
(\%)\end{array}$ & $\begin{array}{c}\mathrm{D} / \mathrm{H}(\mathrm{HDO}) \\
(\%)\end{array}$ & Ref. \\
\hline IRAS 16293-B & $16.6 \pm 1.1$ & $2.4 \pm 0.5$ & $1.8 \pm 0.4$ & $3.9 \pm 1.1$ & $1.2 \pm 0.3$ & $0.046 \pm 0.013$ & {$[1,2,3]$} \\
IRAS 2A & $13.1 \pm 0.7$ & $1.9 \pm 0.5$ & $1.6-7.1$ & $0.8-3.6$ & $1.2 \pm 0.4$ & $0.085 \pm 0.040$ & {$[4,5]$} \\
IRAS 4A & $13.1 \pm 0.7$ & $1.2 \pm 0.4$ & $0.7-3.1$ & $1.2-5.3$ & $0.90 \pm 0.37$ & $0.17 \pm 0.08$ & {$[4,6]$} \\
HH212 & $15.3 \pm 0.7$ & $1.0 \pm 0.3$ & $0.4-2.0$ & $1.5-7.2$ & - & - & {$[7,4]$} \\
NGC 7129 FIRS1 & $19.3 \pm 0.2$ & $0.21 \pm 0.04$ & - & - & - & - & {$[8]$} \\
Sgr B2 & $24 \pm 1$ & 0.04 & 0.07 & 1.7 & 0.13 & - & {$[9]$} \\
Orion KL & $24.3 \pm 0.4$ & $0.037 \pm 0.007$ & $0.15 \pm 0.05$ & $0.73 \pm 0.27$ & - & $0.13 \pm 0.06$ & {$[10,11]$} \\
NGC 6334 IMM1I & $23.9 \pm 0.8$ & $0.020 \pm 0.007$ & $0.48 \pm 0.12$ & $0.13 \pm 0.03$ & - & - & {$[12]$} \\
\hline
\end{tabular}

Notes. For the $\mathrm{CH}_{3} \mathrm{OD}$ column densities in IRAS 2A, IRAS 4A, and $\mathrm{HH} 212$, two estimates are given using the partition functions estimated by Parise (2004) and Jørgensen et al. (2018). The highest $\mathrm{CH}_{3} \mathrm{OD}$ column densities (and the lowest $\left[\mathrm{CH}_{2} \mathrm{DOH}\right] /\left[\mathrm{CH}_{3} \mathrm{OD}\right]$ ratios) are those with the partition function estimated by Jørgensen et al. (2018).

References. [1] Jørgensen et al. (2018); [2] Calcutt et al. (2018); [3] Persson et al. (2013); [4] this work; [5] Coutens et al. (2014); [6] average between $\mathrm{HDO} / \mathrm{H}_{2} \mathrm{O}$ ratios of $5 \times 10^{-3}$ (Taquet et al. 2013a) and $5.4 \times 10^{-4}$ (Persson et al. 2014, M. Persson, priv. comm.); [7] Bianchi et al. (2017); [8] Fuente et al. (2014); [9] Belloche et al. (2016); [10] Peng et al. (2012); [11] Neill et al. (2013); [12] Bøgelund et al. (2018).

fractionation observed in the warm gas-phase of low-mass and massive hot cores would rather reflect the deuteration of methanol formed in interstellar ices within their progenitor dense clouds.

\subsection{Comparison with theoretical predictions}

We compared the observed methanol deuterations with the predictions of the GRAINOBLE astrochemical code by Taquet et al. (2012a, 2013a, 2014), which follows the formation and 
the deuteration of ices in cold dense cores. The code has been extensively described in previous studies and has recently been used to interpret the deuteration towards NGC 6334 measured by Bøgelund et al. (2018). We investigated the effect of the temperature, total density, and time on the methanol deuteration using the same code and same chemical network as in Taquet et al. (2014). However, unlike in Taquet et al. (2014), we ran here a series of pseudo-time-dependent simulations in which the chemistry evolves over time for constant physical properties such as temperature or density. Figure C.1 shows the deuteration of methanol and water in ices as a function of temperature between 10 and $30 \mathrm{~K}$ for three different dense cloud densities, $n_{\mathrm{H}}=10^{4}$, $10^{5}$, and $10^{6} \mathrm{~cm}^{-3}$ at three different times, $0.1 \times t_{\mathrm{FF}}, t_{\mathrm{FF}}$, and $10 \times t_{\mathrm{FF}}$, where $t_{\mathrm{FF}}$ is the free-fall time. $t_{\mathrm{FF}}$ is equal to $4.4 \times 10^{5}$, $1.4 \times 10^{5}$, and $4.4 \times 10^{4} \mathrm{yr}$ at densities of $10^{4}, 10^{5}$, and $10^{6} \mathrm{~cm}^{-3}$, respectively.

The methanol deuteration strongly depends on the density, the considered temperature, and time. Methanol and its deuterated isotopologues are formed through addition reactions of atomic $\mathrm{H}$ and $\mathrm{D}$ on $\mathrm{CO}$ and $\mathrm{H}_{2} \mathrm{CO}$ on ices possibly supplemented by abstraction and substitution reactions (Nagaoka et al. 2005; Hidaka et al. 2007, 2009). The methanol deuteration is therefore governed by the atomic $[\mathrm{D}] /[\mathrm{H}]$ abundance ratio in the gas phase during ice formation. Atomic D is mostly formed through electronic recombination of $\mathrm{H}_{3}^{+}$isotopologues, which in turn are formed through exothermic reactions between $\mathrm{H}_{3}^{+}$ and HD. The efficiency of the backward reaction increases with increasing temperature and with the ortho/para ratio of $\mathrm{H}_{2}$. In addition, reactions between $\mathrm{H}_{3}^{+}$or with its isotopologues and HD are in competition with reactions involving $\mathrm{CO}$. The production of atomic deuterium is therefore enhanced at low temperatures, close to $10 \mathrm{~K}$, when the abundances of $\mathrm{CO}$ and ortho state of $\mathrm{H}_{2}$ are low (see Fig. 1 of Taquet et al. 2012b, for a scheme detailing the gas-phase deuteration network). As a consequence, the strong decrease of up to two orders of magnitudes of the methanol deuteration predicted at $t \geq t_{\mathrm{FF}}$ from 10 to $30 \mathrm{~K}$ is essentially due to the decreased efficiency of deuterium chemistry in the gas phase of cold dense cores.

The observed $\left[\mathrm{CH}_{3} \mathrm{OD}\right] /\left[\mathrm{CH}_{3} \mathrm{OH}\right]$ ratios observed both towards low-mass and massive protostars are relatively well reproduced by the model predictions at $t \geq t_{\mathrm{FF}}$, despite the large dispersion of values among massive protostars. The $\left[\mathrm{CH}_{2} \mathrm{DOH}\right] /\left[\mathrm{CH}_{3} \mathrm{OH}\right]$ abundance ratios of low-mass protostars can also be explained by the models at $t \geq t_{\mathrm{FF}}$, but the massive protostar values can only be explained by a shorter time of $0.1 \times t_{\mathrm{FF}}$ or a low density of $10^{4} \mathrm{~cm}^{-3}$. The predicted $\left[\mathrm{CH}_{2} \mathrm{DOH}\right] /\left[\mathrm{CH}_{3} \mathrm{OD}\right]$ ratio remains close to the statistical value of 3 in most cases and can increase up to $\sim 6$ at $10^{6} \mathrm{~cm}^{-3}, T \sim 10-15 \mathrm{~K}$, and $t=10 \times t_{\mathrm{FF}}$. Our model only focuses on cold surface chemistry and is not able to explain $\left[\mathrm{CH}_{2} \mathrm{DOH}\right] /\left[\mathrm{CH}_{3} \mathrm{OD}\right]$ ratios lower than 1 observed in massive hot cores. $\mathrm{CH}_{3} \mathrm{OD}$ would then need to be produced through other processes such as warm gas-phase chemistry following methanol evaporation within massive hot cores. Osamura et al. (2004) showed that the reaction $\mathrm{H}_{2} \mathrm{DO}^{+}+\mathrm{CH}_{3} \mathrm{OH} \rightarrow \mathrm{CH}_{3} \mathrm{OHD}^{+}+$ $\mathrm{H}_{2} \mathrm{O}$, followed by dissociative recombination, could increase the $\left[\mathrm{CH}_{3} \mathrm{OD}\right] /\left[\mathrm{CH}_{3} \mathrm{OH}\right]$ ratio by a factor of $\sim 5$ at $100 \mathrm{~K}$. However, this increase is obtained at times longer than $10^{5} \mathrm{yr}$ and assuming $[\mathrm{HDO}] /\left[\mathrm{H}_{2} \mathrm{O}\right]$ abundance ratios of $\sim 0.1$ that are about 100 times higher than the observed values. It remains to be tested whether this process could play a major role at higher temperatures and for realistic water deuteration values. On the other hand, hydrogen-deuterium exchanges in warm ices have been proven to be efficient, but only on the hydroxyl functional group of methanol. $\mathrm{CH}_{3} \mathrm{OD}$ would thus give its deuterium to water, inducing a decrease in $\mathrm{CH}_{3} \mathrm{OD}$ abundance in ices before its evaporation (Ratajczak et al. 2009; Faure et al. 2015).

Unlike methanol, water does not show any significant decrease of its observed deuteration with the dust temperature because it remains around $0.1 \%$ between 13 and $24 \mathrm{~K}$. The low water deuteration has been interpreted as due to an early formation of solid water in the translucent phase at low visual extinctions, when the dark cloud is still lukewarm, with temperatures of 15-20 K, and when the abundances of $\mathrm{CO}$ and ortho $\mathrm{H}_{2}$ are still high (Taquet et al. 2013a, 2014). Methyl cyanide is thought to be mostly produced either on warm ices from recombination between the $\mathrm{CN}$ and $\mathrm{CH}_{3}$ radicals or in the gas phase through the radiative association between $\mathrm{HCN}$ and $\mathrm{CH}_{3}^{+}$, after the prestellar stage. Comparing the methyl cyanide deuteration with the model predictions with constant physical conditions of cold cores is therefore not relevant.

\subsection{Deuteration from low-mass protostars to comets}

Solar System comets mostly contain pristine material that has poorly evolved since the end of the Solar System formation 4.5 billions years ago. Their chemical composition should reflect the chemical composition of the solar nebula (see Mumma \& Charnley 2011, for instance). Comparing the deuteration of water and organics measured around young solar-type protostars on Solar System scales and in Solar System comets should allow us to follow the chemical evolution throughout the star formation process.

Figure 8 compares the (statistical) deuterium fractionation of methanol, methyl cyanide, and water observed in low-mass protostars with the deuteration of $\mathrm{HCN}$ in protoplanetary discs and of $\mathrm{CH}_{3} \mathrm{OH}, \mathrm{HCN}$, and water in comets. Deuteration of water has been estimated in four nearby protostars on Solar System scales by Taquet et al. (2013a), Persson et al. (2014) and Coutens et al. (2014) through interferometric observations of HDO and $\mathrm{H}_{2}^{18} \mathrm{O}$ transitions and about a dozen comets (see the list of references in the caption of Fig. 7). Water deuteration in protostars is only higher by about a factor of two than the deuteration measured in comets. Unlike water, cometary deuterated methanol has not been detected so far. The most stringent upper limit in the methanol deuteration has been given by Crovisier et al. (2004) towards comet Hale-Bopp, with a $\left[\mathrm{CH}_{2} \mathrm{DOH}\right] /\left[\mathrm{CH}_{3} \mathrm{OH}\right]$ abundance ratio lower than $0.8 \%$. The $\left[\mathrm{CH}_{2} \mathrm{DOH}\right] /\left[\mathrm{CH}_{3} \mathrm{OH}\right]$ ratio of 3-6\% estimated with interferometers towards young protostars is higher by a factor of $4-8$ than the estimate made in comet Hale-Bopp. A low methanol deuteration in comets remains to be confirmed with a clear detection of deuterated methanol, either through the analysis of the high-resolution ROSINA mass spectrometer data at mass 33 on board the Rosetta space probe (see for instance Le Roy et al. 2015), or through future sensitive submillimeter or millimeter ground-based surveys of nearby and bright comets using interferometers like ALMA or NOEMA.

Assuming that the observed deuteration towards nearby lowmass protostars is representative for the deuteration of the young Sun, this comparison suggests that an important reprocessing of the organic material occurred in the solar nebula whilst little water reprocessing in the solar nebula is required. Three reasons can be suggested. First, methanol observed in the hot core of protostars on $\sim 100$ AU scales is reprocessed before entering in the disc mid-plane through high-temperature chemistry involving reactions with $\mathrm{H}$ or $\mathrm{OH}$ because they have moderate energy barriers of 2200-3000 K ( $\mathrm{Li} \&$ and Williams 1996). However, the temperature of the hot corino is likely too low to trigger 


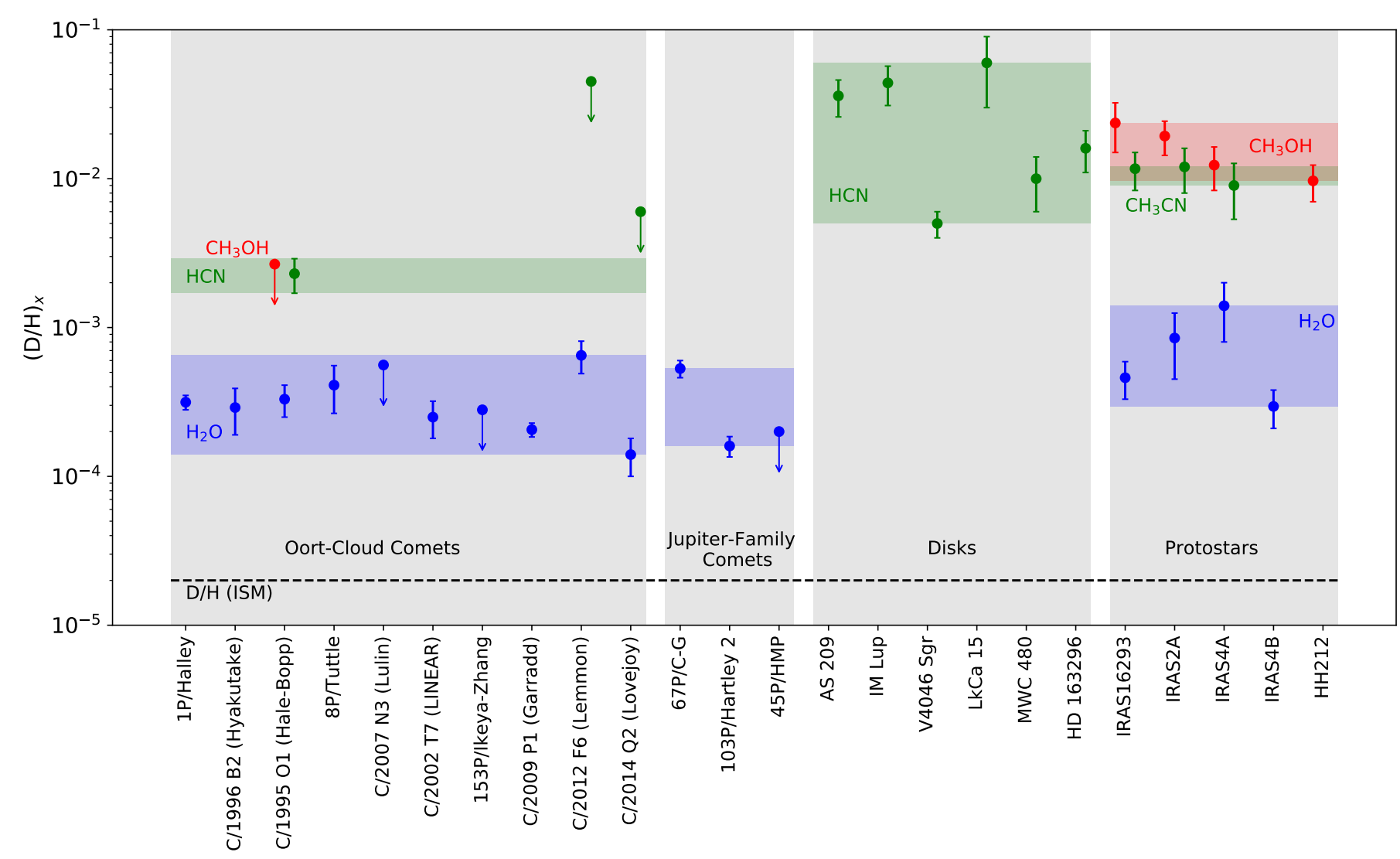

Fig. 8. Statistical D/H ratios in Solar System comets, protoplanetary discs, and low-mass Class 0 protostars for methanol (red), water (blue), and HCN or $\mathrm{CH}_{3} \mathrm{CN}$ (green). References: 1P/Halley: Eberhardt et al. (1995); C/1996 B2 (Hyakutake): Bockelée-Morvan et al. (1998); C/1995 O1 (Hale-Bopp): Meier et al. (1998); Crovisier et al. (2004); 8P/Tuttle: Villanueva et al. (2009); C/2007 N3 (Lulin): Gibb et al. (2012); C/2002 T7 (LINEAR): Hutsemékers et al. (2008); 153P/lkeya-Zhang: Biver et al. (2006); C/2009 P1 (Garradd): Bockelée-Morvan et al. (2012); C/2012 F6 (Lemmon): Biver et al. (2016); C/2014 Q2 (Lovejoy): Biver et al. (2016); 67P/C-G: Altwegg et al. (2015); 103P/Hartley 2: Hartogh et al. (2011); 45P/HMP: Lis et al. (2013); All discs: Huang et al. (2017); IRAS 16293: Persson et al. (2014); Jørgensen et al. (2018); Calcutt et al. (2018); IRAS 2A: Coutens et al. (2014), this work; IRAS 4A: average values derived by Taquet et al. (2013b) and Persson et al. (2014, M. Persson priv. comm.), this work; IRAS4B: Persson et al. (2014).

such reactions and other processes that increase the gas temperature to $\sim 1000 \mathrm{~K}$, such as accretion shocks that form at the interface of the envelope and the disc in formation (Aota et al. 2015), would be needed. Second, physical processes such as turbulence in the disc could transport the material from the disc mid-plane to the atmosphere where efficient photolytic processes can photo-dissociate organic molecules. Albertsson et al. (2014) and Furuya et al. (2014) demonstrated that radial and vertical turbulence can decrease the water deuteration by typically one order of magnitude in the cometary zone. However, it is yet to be demonstrated that this process could also affect the deuteration of more complex organic species with slightly different chemistries. A third possibility is that the cloud at the origin of the Solar System was initially slightly warmer than the temperatures of nearby dark clouds in which the observed protostars are located. This has recently been suggested by Taquet et al. (2016), who concluded that a dark cloud temperature of $\sim 20 \mathrm{~K}$, together with a dark cloud density higher than $10^{5} \mathrm{~cm}^{-3}$, was needed to explain the high abundance of $\mathrm{O}_{2}$ and its strong correlation with water in comet $67 \mathrm{P} / \mathrm{CG}$. This modelling work confirmed the studies of meteoritic data, which suggest that the Solar System was born in a dense cluster of stars (see Adams 2010). According to Fig. 7, an increase in dark cloud temperature from 10 to $20 \mathrm{~K}$ would decrease the methanol deuteration from 2-6 to $0.2-0.6 \%$, depending on the density, which seems to be in good agreement with the value found in comet 67P/CG.

\section{Conclusions}

We analysed several existing observational datasets obtained with the PdBI and ALMA sub-millimeter interferometers to estimate the methanol deuterations in the hot cores surrounding three protostars on Solar System scales. For this purpose, we analysed several dozen deuterated methanol transitions with a PD analysis in order to measure the $\left[\mathrm{CH}_{2} \mathrm{DOH}\right] /\left[\mathrm{CH}_{3} \mathrm{OH}\right]$ and $\left[\mathrm{CH}_{3} \mathrm{OD}\right] /\left[\mathrm{CH}_{3} \mathrm{OH}\right]$ abundance ratios. The obtained $\left[\mathrm{CH}_{2} \mathrm{DOH}\right] /\left[\mathrm{CH}_{3} \mathrm{OH}\right]$ ratios of $3-6 \%$ and $\left[\mathrm{CH}_{3} \mathrm{OD}\right] /\left[\mathrm{CH}_{3} \mathrm{OH}\right]$ ratios of $0.4-1.6 \%$ are typically one order of magnitude lower than previous estimates derived from single-dish observations towards the same sources, and are in good agreement with the recent ALMA measurements by Jørgensen et al. (2018) towards the low-mass protostar IRAS 16293-B. We then compared our methanol deuteration estimates with previous measurements of intermediate- and high-mass hot cores with similar observational properties and analysis methods. Unlike water, which does not show strong variation of its deuteration between low-mass and high-mass protostars, we find that the methanol deuteration around massive hot cores is much lower by one to two orders of magnitude. This strong difference could be attributed to a different physical or chemical history of the sources. Methanol observed around protostars is mostly formed in interstellar ices during the previous molecular cloud phase. Dust temperature maps derived with the Herschel or Planck space observatories 
suggest that the observed massive protostars were born in warm molecular clouds of $T \sim 24 \mathrm{~K}$, whilst low-mass protostars are located in more quiescent and cold regions with temperatures lower than $15 \mathrm{~K}$. Comparing the observed deuteration values with the predictions of the GRAINOBLE astrochemical model on ice formation and deuteration, an increase of $10 \mathrm{~K}$ in the dust temperature is enough to explain a decrease of the observed methanol deuteration, depending on the density and the chemical time. Finally, the methanol deuterations measured towards young solar-type protostars at high angular resolution on Solar System scales seem to be higher by a factor of $\sim 5$ than the upper limit in methanol deuteration estimated in comet Hale-Bopp by Crovisier et al. (2004). If this result is confirmed by subsequent observations of other comets, it would imply that an important reprocessing of the organic material likely occurred in the solar nebula during the formation of the Solar System.

Acknowledgements. This work is based on observations carried out with the ALMA Interferometer under project numbers ADS/JAO.ALMA\#2012.1.00997.S and ADS/JAO.ALMA\#2016.1.01475.S data (PI: C. Codella) and with the IRAM PdBI/NOEMA Interferometer under project numbers V05B and V010 (PI: M.V. Persson) and U003 (PI: V. Taquet). ALMA is a partnership of ESO (representing its member states), NSF (USA) and NINS (Japan), together with NRC (Canada) and NSC and ASIAA (Taiwan), in cooperation with the Republic of Chile. The Joint ALMA Observatory is operated by ESO, AUI/NRAO and NAOJ. IRAM is supported by INSU/CNRS (France), MPG (Germany) and IGN (Spain). V.T acknowledges the financial support from the European Union's Horizon 2020 research and innovation programme under the Marie Sklodowska-Curie grant agreement n. 664931. E.B., Ce.Ce., C.K., A.L. and Cl.Co acknowledge the funding from the European Research Council (ERC) under the European Union's Horizon 2020 research and innovation programme, for the Project "The Dawn of Organic Chemistry" (DOC), grant agreement No 741002. Cl.Co acknowledges the funding from PRIN-INAF 2016 "The Cradle of Life - GENESIS-SKA (General Conditions in Early Planetary Systems for the rise of life with SKA)". Ce.Ce. and Cl.Co acknowledge the financial support from the European MARIE SKŁODOWSKA-CURIE ACTIONS under the European Union's Horizon 2020 research and innovation programme, for the Project "Astro-Chemistry Origins" (ACO), Grant No 811312.

\section{References}

Adams, F. C. 2010, ARA\&A, 48, 47

Albertsson, T., Semenov, D., \& Henning, T. 2014, A\&A, 784, A39

Altwegg, K., Balsiger, H., Bar-Nun, A., et al. 2015, Science, 47, 27

Anderson, T., Crownover, R. L., Herbst, E., \& De Lucia, F. C. 1988, ApJS, 67, 135

André, P., Men'shchikov, A., Bontemps, S., et al. 2010, A\&A, 518, L102

Aota, T., Inoue, T., \& Aikawa, Y. 2015, A\&A, 799, A141

Belloche, A., Müller, H. S. P., Garrod, R. T., \& Menten, K. M. 2016, A\&A, 587, A91

Bianchi, E., Codella, C., Ceccarelli, C., et al. 2017, A\&A, 606, L7

Biver, N., Bockelée-Morvan, D., Crovisier, J., et al. 2006, A\&A, 449, 1255

Biver, N., Moreno, R., Bockelée-Morvan, D., et al. 2016, A\&A, 589, A78

Bockelée-Morvan, D., Gautier, D., Lis, D. C., et al. 1998, Icarus, 133, 147

Bockelée-Morvan, D., Biver, N., Swinyard, B., et al. 2012, A\&A, 544, L15

Bøgelund, E., McGuire, M. A., Ligterink, N. F. W., et al. 2018, A\&A, 615, A88

Calcutt, H., Jørgensen, J. K., Müller, H. S. P., et al. 2018, A\&A, 616, A90

Caselli, P., \& Ceccarelli, C. 2012, A\&ARv, 20, 56

Caux, E., Kahane, C., Castets, A., et al. 2011, A\&A, 532, A23

Cazaux, S., Tielens, A. G. G. M., Ceccarelli, C., et al. 2003, ApJ, 593, L51

Cazaux, S., Caselli, P., \& Spaans, M. 2011, ApJ, 741, L34

Ceccarelli, C., Castets, A., Loinard, L., Caux, E., \& Tielens, A. G. G. M. 1998 A\&A, 338, L43

Ceccarelli, C., Caselli, P., Bockelée-Morvan, D., et al. 2014, Protostars and Planets VI (Tucson, AZ: University of Arizona Press), 859

Charnley, S. B., Tielens, A. G. G. M., Rodgers, S. D., et al. 1997, ApJ, 482, L203

Codella, C., Ceccarelli, C., Lee, C.-F., et al. 2019, Earth Space Chem., 3, 2110

Coutens, A., Vastel, C., Caux, E., et al. 2012, A\&A, 539, A132

Coutens, A., Jørgensen, J. K., Persson, M. V., et al. 2018, ApJ, 792, L5

Crovisier, J., Bockelée-Morvan, D., Colom, P., et al. 2004, A\&A, 418, 1141

De Simone, M., Codella, C., Testi, L., et al. 2017, A\&A, 599, A121

Duan, Y.-B., Ozier, I., Tsunekawa, S., \& Takagi, K. 2003, J. Mol. Spectr., 218 95
Eberhardt, P., Reber, M., Krankowsky, D., \& Hodges, R. R. 1995, A\&A, 302, 301

Etxaluze, M., Goicoechea, J. R., Cernicharo, J., et al. 2013, A\&A, 556, A137

Faure, A., Faure, M., Theulé, E., Quirico, E., \& Schmitt, B. 2015, A\&A, 584 A98

Fuente, A., Cernicharo, J., Caselli, P., et al. 2014, A\&A, 568, A65

Furuya, K., \& Aikawa, Y. 2014, ApJ, 790, 97

Garrod, R., Park, I. H., Caselli, P., \& Herbst, E. 2006, Faraday Discuss., 133, 51

Geppert, W. D., Hamberg, M., Thomas, R. D. et al. 2006, Faraday Discuss., 133, 177

Gibb, E. L., Bonev, B. P., Villanueva, G., et al. 2012, ApJ, 750, 102

Goldsmith, P. F., Langer, W. D., \& Velusamy, T. 1999, ApJ, 519, L173

Hartogh, P., Lis, D. C., Bockelée-Morvan, D., et al. 2011, Nature, 478, 218

Herbst, E., \& van Dishoeck, E. F. 2009, ARA\&A, 47, 427

Hidaka, H., Kouchi, A., \& Watanabe, N. 2007, J. Chem. Phys., 126, 204707

Hidaka, H., Watanabe N., \& Kouchi, A. 2009, ApJ, 702, 291

Hirota, T., Bushimata, T., Choi, Y. K., Honma, M., et al. 2008, PASJ, 60, 37

Huang, J., Öberg, K. I., Qi, C., Aikawa, Y., et al. 2017, ApJ, 835, 231

Hutsemékers, D., Manfroid, J., Jehin, E., Zucconi, J.-M., \& Arpigny, C. 2008, A\&A, 490, L31

Jørgensen, J. K., Lahuis, F., Schöier, F. L., et al. 2005, ApJ, 631, L77

Jørgensen, J. K., Bourke, T. L., Nguyen Luong, Q., \& Takakuwa, S. 2011, A\&A, $534, \mathrm{~A} 100$

Jørgensen, J. K., van der Wiel, M. H. D., Coutens, A., et al. 2016, A\&A, 595, A117

Jørgensen, J. K., Müller, H. S. P., Calcutt, H., et al. 2018, A\&A, 620, A170

Kounkel, M., Hartmann, L., Loinard, L., Ortiz-León, G. N. et al. 2017, ApJ, 834, 142

Lauvergnat, D., Coudert, L. H., Klee, S., \& Smirnov, M. 2009, J. Mol. Spectr., 256,204

Lee, C.-F., Li, Z.-Y., Ho, P. T. P., 2017, ApJ, 843, 27

Le Roy, L., Altwegg, K., Balsiger, H., et al. 2015, A\&A, 583, A1

Li, S. C., Williams, F. A. 1996, Symp. ( Intl.) Combust., 26, 1017

Linsky, J. L. 2003, Space Sci. Rev., 106, 49

Lis, D. C., Biver, N., Bockelée-Morvan, D., et al. 2013, ApJ, 774, L3

Looney, L. W., Mundy, L. G., \& Welch, W. J. 2000, ApJ, 529, 477

López-Sepulcre, A., Sakai, N., Neri, R., et al. 2017, A\&A, 606, A121

Maury, A. J., Belloche, A., André, P., et al., S. 2017, A\&A, 563, L2

Meier, R., Owen, T. C., Matthews, H. E., et al. 1998, Science, 279, 842

Molinari, S., Swinyard, B., Bally, J., et al. 2010, PASP, 122, 314

Motte, F., Zavagno, A., Bontemps, S., et al. 2010, A\&A, 518, L77

Mumma, M., \& Charnley, S. B. 2011, ARA\&A, 49, 471

Nagaoka, A., Watanabe, N., \& Kouchi, A. 2005, ApJ, 624, L29

Nguyen, L., Walters, A., Margulès, L., et al. 2013, A\&A, 553, A84

Neill, J. L., Wang, S., Bergin, E. A., et al. 2013, ApJ, 770, 142

Osamura, Y., Roberts, H., \& Herbst, E. 2004, A\&A, 421, 1101

Parise, B. PhD Thesis, Université Toulouse III, France

Parise, B., Ceccarelli, C., Tielens, A. G. G. M., et al. 2002, A\&A, 393, L49- L53

Parise, B., Ceccarelli, C., Tielens, A. G. G. M., et al. 2006, A\&A, 453, 949

Pearson, J. C., Yu, S., \& Drouin, B. J. 2012, J. Mol. Spectr., 280, 119

Peng, T.-C., Despois, D., Brouillet, N., Parise, B., \& Baudry, A. 2012, A\&A, 543, A 152

Persson, M. V., Jørgensen, J. K., \& van Dishoeck, E. F. 2012, A\&A, 541, A39

Persson, M. V., Jørgensen, J. K., \& van Dishoeck, E. F. 2013, A\&A, 549, L3

Persson, M. V., Jørgensen, J. K., van Dishoeck, E. F., \& Harsono, D. 2014, A\&A, 563, A74

Ratajczak, A., Quirico, E., Faure, A., Schmitt, B., \& Cecarelli, C. 2009, A\&A, 496, L21

Ratajczak, A., Taquet, V., \& Kahane, C., et al. 2011, A\&A, 528, L13

Rimola, A., Taquet, V., \& Ugliengo, P. 2014, A\&A, 572, A70

Russeil, D., Schneider, N., Anderson, L. D., et al. 2013, A\&A, 554, A42

Taquet, V., Ceccarelli, C., \& Kahane, C. 2012a, A\&A, 538, A42

Taquet, V., Ceccarelli, C., \& Kahane, C. 2012b, ApJ, 748, L3

Taquet, V., Peters, P., Kahane, C., et al. 2013a, A\&A, 550, A127

Taquet, V., López-Sepulcre, A., Ceccarelli, C., et al. 2013b, ApJ, 768, L29

Taquet, V., Charnley, S. B., \& Sipilä, O. 2014, ApJ, 791, 1

Taquet, V., López-Sepulcre, A., Ceccarelli, C., et al. 2015, ApJ, 804, 81

Taquet, V., Furuya, K., Walsh, C., \& van Dishoeck, E. F. 2016, MNRAS, 462, S99

Tigé, J., Motte, F., Russeil, D., et al. 2017, A\&A, 602, A77

van Dishoeck, E. F., Blake, G. A., Jansen, D. J., \& Groesbeck, T. D. 1995, ApJ, 447, 760

Villanueva, G. L., Mumma, M. J., Bonev, B. P., et al. 2009, ApJ, 690, L5

Watanabe, N., \& Kouchi, A. 2002, ApJ, 571, L173

Wilson, T. L., \& Rood, R. T. 1994, ARA\&A, 32, 191

Zucker, C., Schlafly, E. F., Speagle, J. S., et al. 2018, ApJ, 869, 83 


\section{Appendix A: Line parameters of the targeted transitions}

Table A.1. Line parameters of $\mathrm{CH}_{2} \mathrm{DOH}$ lines observed towards IRAS 2A and IRAS 4A.

\begin{tabular}{|c|c|c|c|c|c|c|}
\hline Number & $\begin{array}{c}\text { Frequency } \\
(\mathrm{GHz})\end{array}$ & Transition & $\begin{array}{l}E_{\mathrm{up}} \\
(\mathrm{K})\end{array}$ & $\begin{array}{c}A_{\mathrm{ul}} \\
\left(\mathrm{s}^{-1}\right)\end{array}$ & $\begin{array}{c}\text { Flux (IRAS 2A) } \\
\left(\mathrm{Jy} \mathrm{km} \mathrm{s}^{-1}\right)\end{array}$ & $\begin{array}{l}\text { Flux (IRAS 4A) } \\
\qquad\left(\mathrm{Jy} \mathrm{km} \mathrm{s}^{-1}\right) \\
\end{array}$ \\
\hline 1 & 166.063164 & $2_{2,0} e_{1}-1_{1,0} o_{1}$ & 33.0 & $2.40(-5)$ & $0.285 \pm 0.081$ & $0.199 \pm 0.076$ \\
\hline 2 & 166.787448 & $2_{2,1} e_{1}-1_{1,1} o_{1}$ & 33.0 & $2.43(-5)$ & $0.292 \pm 0.071$ & $0.184 \pm 0.051$ \\
\hline 3 & 225.878232 & $3_{1,3} o_{1}-2_{0,2} o_{1}$ & 35.6 & $3.23(-5)$ & $0.607 \pm 0.123$ & $0.204 \pm 0.056$ \\
\hline 4 & 226.818248 & $5_{1,4} e_{0}-4_{1,3} e_{0}$ & 36.7 & $3.58(-5)$ & $0.931 \pm 0.187$ & $0.349 \pm 0.074$ \\
\hline 5 & 144.762399 & $4_{1,3} e_{1}-3_{0,3} o_{1}$ & 38.2 & $8.80(-6)$ & $0.132 \pm 0.028$ & $0.153 \pm 0.043$ \\
\hline 6 & 164.108467 & $5_{0,5} e_{1}-4_{1,4} e_{1}$ & 45.6 & $2.67(-6)$ & $0.086 \pm 0.031$ & $0.090 \pm 0.031$ \\
\hline 7 & 223.898819 & $3_{2,2} o_{1}-4_{2,2} e_{1}$ & 48.3 & $1.56(-6)$ & $0.450 \pm 0.095$ & $0.219 \pm 0.068$ \\
\hline 8 & 225.667709 & $5_{1,4} e_{1}-4_{1,3} e_{1}$ & 49.0 & $4.44(-5)$ & $0.272 \pm 0.062$ & $0.351 \pm 0.075$ \\
\hline 9 & 224.928016 & $5_{1,4} e_{2}-4_{1,3} e_{2}$ & 55.3 & $4.42(-5)$ & $1.390 \pm 0.280$ & $0.428 \pm 0.092$ \\
\hline 10 & 144.134719 & $5_{3,2} e_{0}-6_{2,5} e_{0}$ & 68.1 & $2.64(-6)$ & $0.069 \pm 0.026$ & $0.098 \pm 0.035$ \\
\hline 11 & 223.691457 & $5_{3,3} e_{0}-4_{3,2} e_{0}$ & 68.1 & $2.17(-5)$ & $0.601 \pm 0.122$ & $0.203 \pm 0.043$ \\
\hline 12 & 223.697110 & $5_{3,2} e_{0}-4_{3,1} e_{0}$ & 68.1 & $2.17(-5)$ & $0.608 \pm 0.124$ & $0.217 \pm 0.051$ \\
\hline 13 & 225.848108 & $7_{0,7} o_{1}-6_{1,6} o_{1}$ & 78.3 & $2.17(-5)$ & $0.604 \pm 0.123$ & $0.540 \pm 0.114$ \\
\hline 14 & 165.861895 & $8_{0,8} e_{1}-81,7 e_{0}$ & 90.4 & $8.99(-6)$ & $0.217 \pm 0.057$ & $0.056 \pm 0.020$ \\
\hline 15 & 164.577869 & $8_{1,7} e_{1}-7_{2,6} e_{1}$ & 94.4 & $1.52(-6)$ & $0.058 \pm 0.023$ & $0.071 \pm 0.029$ \\
\hline \multirow[t]{2}{*}{16} & 223.616196 & $5_{4,2} e_{0}-4_{4,1} e_{0}$ & 95.1 & $1.26(-5)$ & $0.522 \pm 0.107$ & $0.264 \pm 0.065$ \\
\hline & 223.616210 & $5_{4,1} e_{0}-4_{4,0} e_{0}$ & 95.1 & $1.26(-5)$ & & \\
\hline 17 & 164.374775 & $10_{4,7} e_{0}-10_{3,7} o_{1}$ & 181.0 & $5.91(-6)$ & $0.073 \pm 0.029$ & $0.062 \pm 0.031$ \\
\hline 18 & 164.388327 & $10_{4,6} e_{0}-10_{3,8} o_{1}$ & 181.0 & $5.91(-6)$ & $0.087 \pm 0.032$ & $0.106 \pm 0.043$ \\
\hline 19 & 143.566661 & $12_{2,10} e_{0}-11_{3,9} e_{0}$ & 184.5 & $3.76(-6)$ & $0.082 \pm 0.027$ & $0.041 \pm 0.016$ \\
\hline 20 & 224.273919 & $10_{5,6} e_{0}-11_{4,7} e_{0}$ & 215.4 & $1.16(-5)$ & $0.126 \pm 0.035$ & $0.082 \pm 0.025$ \\
\hline 21 & 224.285457 & $10_{5,5} e_{0}-11_{4,8} e_{0}$ & 215.4 & $1.16(-5)$ & $0.178 \pm 0.041$ & $0.152 \pm 0.038$ \\
\hline 22 & 166.950015 & $12_{4,9} e_{0}-12_{3,9} o_{1}$ & 230.4 & $6.80(-6)$ & $0.079 \pm 0.031$ & $0.065 \pm 0.018$ \\
\hline 23 & 166.995876 & $12_{4,8} e_{0}-12_{3,10} o_{1}$ & 230.4 & $6.80(-6)$ & $0.072 \pm 0.027$ & $0.064 \pm 0.021$ \\
\hline 24 & 142.838827 & $16_{1,15} \circ 1-16_{0,16} \circ 1$ & 316.2 & $9.85(-6)$ & $0.138 \pm 0.031$ & $0.099 \pm 0.026$ \\
\hline 25 & 225.551640 & $17_{2,15} \circ 1-17_{1,16} \circ 1$ & 363.6 & $3.28(-5)$ & $0.273 \pm 0.059$ & $0.149 \pm 0.042$ \\
\hline
\end{tabular}

Table A.2. Line parameters of $\mathrm{CH}_{3} \mathrm{OD}$ lines observed towards IRAS 2A and IRAS 4A.

\begin{tabular}{lcccccc}
\hline \hline Number & $\begin{array}{c}\text { Frequency } \\
(\mathrm{GHz})\end{array}$ & Transition & $\begin{array}{c}E_{\mathrm{up}} \\
(\mathrm{K})\end{array}$ & $\begin{array}{c}A_{\mathrm{ul}} \\
\left(\mathrm{s}^{-1}\right)\end{array}$ & $\begin{array}{c}\text { Flux (IRAS 2A) } \\
\left(\mathrm{Jy} \mathrm{km} \mathrm{s}^{-1}\right)\end{array}$ & $\begin{array}{c}\text { Flux (IRAS 4A) } \\
\left(\mathrm{Jy} \mathrm{km} \mathrm{s}^{-1}\right)\end{array}$ \\
\hline 1 & 226.53867 & $5_{0+}-4_{0+} \mathrm{A}$ & 32.7 & $4.31(-5)$ & $0.869 \pm 0.175$ & $0.290 \pm 0.064$ \\
2 & 226.350191 & $5_{0}-4_{0} \mathrm{E}$ & 36.4 & $4.66(-5)$ & $0.744 \pm 0.151$ & $0.380 \pm 0.084$ \\
3 & 226.185930 & $5_{-1}-4_{-1} \mathrm{E}$ & 37.3 & $4.16(-5)$ & $0.433 \pm 0.088$ & $0.206 \pm 0.051$ \\
4 & 143.741650 & $5_{1-}-5_{0+} \mathrm{A}$ & 39.6 & $3.52(-5)$ & $0.237 \pm 0.054$ & $0.121 \pm 0.029$ \\
5 & 226.92258 & $5_{-2}-4_{-2} \mathrm{E}$ & 50.2 & $3.71(-5)$ & $0.355 \pm 0.074$ & $0.143 \pm 0.035$ \\
6 & 226.892864 & $5_{2}-4_{2} \mathrm{E}$ & 54.4 & $3.71(-5)$ & $0.267 \pm 0.055$ & $0.113 \pm 0.025$ \\
7 & 226.94283 & $5_{2+}-4_{2+} \mathrm{A}$ & 54.4 & $3.70(-5)$ & $0.436 \pm 0.091$ & $0.253 \pm 0.067$ \\
8 & 226.825536 & $5_{3}-4_{3} \mathrm{E}$ & 70.5 & $3.71(-5)$ & $0.254 \pm 0.053$ & $0.119 \pm 0.026$ \\
9 & 226.70660 & $5_{2-}-4_{2-} \mathrm{A}$ & 100 & $2.84(-5)$ & $0.300 \pm 0.062$ & $0.149 \pm 0.040$ \\
10 & 226.73886 & $5_{-4}-4_{-4} \mathrm{E}$ & 104.3 & $1.60(-5)$ & $0.073 \pm 0.022$ & $0.062 \pm 0.031$ \\
\hline
\end{tabular}

Table A.3. Line parameters of $\mathrm{CHD}_{2} \mathrm{OH}$ lines observed towards IRAS 2A and IRAS 4A.

\begin{tabular}{lcccccc}
\hline \hline Number & $\begin{array}{c}\text { Frequency } \\
(\mathrm{GHz})\end{array}$ & Transition & $\begin{array}{c}E_{\mathrm{up}} \\
(\mathrm{K})\end{array}$ & $\begin{array}{c}A_{\mathrm{ul}} \\
\left(\mathrm{s}^{-1}\right)\end{array}$ & $\begin{array}{c}\text { Flux (IRAS 2A) } \\
\left(\mathrm{Jy} \mathrm{km} \mathrm{s}^{-1}\right)\end{array}$ & $\begin{array}{c}\text { Flux (IRAS 4A) } \\
\left(\mathrm{Jy} \mathrm{km} \mathrm{s}^{-1}\right)\end{array}$ \\
\hline 1 & 166.435 & $4_{0}-3_{0} e_{0}$ & 20.0 & $1.67(-5)$ & $0.245 \pm 0.066$ & $0.151 \pm 0.028$ \\
2 & 166.327 & $4_{0}-3_{0} o_{1}$ & 28.8 & $1.67(-5)$ & $0.248 \pm 0.107$ & $0.129 \pm 0.030$ \\
3 & 166.234 & $4_{0}-3_{0} e_{1}$ & 38.4 & $1.66(-5)$ & $0.272 \pm 0.064$ & $0.139 \pm 0.033$ \\
4 & 166.271 & $4_{2-}-3_{2-} e_{1}$ & 51.3 & $1.25(-5)$ & $0.184 \pm 0.066$ & $0.106 \pm 0.026$ \\
5 & 166.304 & $4_{2+}-3_{2+} e_{1}$ & 51.3 & $1.25(-5)$ & $0.147 \pm 0.045$ & $0.074 \pm 0.021$ \\
6 & 166.297 & $4_{3+}-3_{3-} e_{1}$ & 67.0 & $7.14(-6)$ & $0.176 \pm 0.055$ & $0.048 \pm 0.020$ \\
& 166.298 & $4_{3-}-3_{3-} e_{1}$ & 67.0 & $7.14(-6)$ & & \\
\hline
\end{tabular}


Table A.4. Line parameters of $\mathrm{CH}_{2} \mathrm{DCN}$ lines observed towards IRAS $2 \mathrm{~A}$ and IRAS 4A.

\begin{tabular}{lcccccc}
\hline \hline Number & $\begin{array}{c}\text { Frequency } \\
(\mathrm{GHz})\end{array}$ & Transition & $\begin{array}{c}E_{\mathrm{up}} \\
(\mathrm{K})\end{array}$ & $\begin{array}{c}A_{\mathrm{ul}} \\
\left(\mathrm{s}^{-1}\right)\end{array}$ & $\begin{array}{c}\text { Flux (IRAS 2A) } \\
\left(\mathrm{Jy} \mathrm{km} \mathrm{s}^{-1}\right)\end{array}$ & $\begin{array}{c}\text { Flux (IRAS 4A) } \\
\left(\mathrm{Jy} \mathrm{km} \mathrm{s}^{-1}\right)\end{array}$ \\
\hline 1 & 224.754530 & $13_{1,13}-12_{1,12}$ & 80.9 & $9.72(-4)$ & $0.159 \pm 0.032$ & $0.081 \pm 0.030$ \\
2 & 225.723769 & $13_{3,11}-13_{3,10}$ & 124.4 & $9.38(-4)$ & $0.130 \pm 0.030$ & $0.089 \pm 0.018$ \\
3 & 225.724053 & $13_{3,10}-13_{3,9}$ & 124.4 & $9.38(-4)$ & $0.130 \pm 0.030$ & $0.089 \pm 0.018$ \\
4 & 225.726540 & $13_{3,12}-13_{3,11}$ & 97.4 & $9.68(-4)$ & $0.162 \pm 0.037$ & $0.069 \pm 0.018$ \\
5 & 225.781540 & $13_{2,11}-13_{2,10}$ & 97.4 & $9.98(-4)$ & $0.162 \pm 0.037$ & $0.069 \pm 0.020$ \\
\hline
\end{tabular}

Table A.5. Line parameters of $\mathrm{CH}_{2} \mathrm{DOH}$ lines observed towards $\mathrm{HH} 212$.

\begin{tabular}{lccccc}
\hline \hline Number & $\begin{array}{c}\text { Frequency } \\
(\mathrm{GHz})\end{array}$ & Transition & $\begin{array}{c}E_{\text {up }} \\
(\mathrm{K})\end{array}$ & $\begin{array}{c}A_{\mathrm{ul}} \\
\left(\mathrm{s}^{-1}\right)\end{array}$ & $\begin{array}{c}\text { Flux (HH212) } \\
\left.(\mathrm{Jy} \mathrm{km} \mathrm{s})^{-1}\right)\end{array}$ \\
\hline 1 & 348.16076 & $4_{1,3} e_{1}-4_{0,4} e_{0}$ & 38.1 & $2.03(-4)$ & $0.152 \pm 0.031$ \\
2 & 338.95711 & $6_{1,6} e_{0}-5_{0,5} e_{0}$ & 48.4 & $1.59(-4)$ & $0.157 \pm 0.031$ \\
3 & 337.34866 & $9_{0,9} e_{0}-8_{1,8} o_{0}$ & 96.3 & $1.46(-4)$ & $0.159 \pm 0.032$ \\
4 & 350.09024 & $5_{4,2} e_{1}-5_{3,2} o_{1}$ & 104.2 & $7.58(-5)$ & $0.065 \pm 0.065$ \\
5 & 350.09038 & $5_{4,1} e_{1}-5_{3,3} o_{1}$ & 104.2 & $7.58(-5)$ & $0.065 \pm 0.065$ \\
6 & 350.02735 & $6_{4,3} e_{1}-6_{3,3} o_{1}$ & 117.1 & $9.06(-5)$ & $0.078 \pm 0.016$ \\
7 & 350.02777 & $6_{4,2} e_{1}-6_{3,4} o_{1}$ & 117.1 & $9.06(-5)$ & $0.078 \pm 0.016$ \\
8 & 349.95168 & $7_{4,4} e_{1}-7_{3,4} o_{1}$ & 132.1 & $1.00(-4)$ & $0.201 \pm 0.040$ \\
9 & 338.86898 & $13_{1,12} e_{0}-12_{0,12} o_{1}$ & 201.8 & $2.81(-5)$ & $0.080 \pm 0.016$ \\
10 & 347.76728 & $18_{4,15} e_{1}-18_{3,15} o_{1}$ & 438.2 & $1.31(-4)$ & $0.046 \pm 0.009$ \\
11 & 347.95281 & $18_{4,14} e_{1}-18_{3,16} o_{1}$ & 438.2 & $1.31(-4)$ & $0.057 \pm 0.011$ \\
\hline
\end{tabular}

Table A.6. Line parameters of the $\mathrm{CH}_{3} \mathrm{OD}$ line observed towards $\mathrm{HH} 212$.

\begin{tabular}{lccccc}
\hline \hline Number & $\begin{array}{c}\text { Frequency } \\
(\mathrm{GHz})\end{array}$ & Transition & $\begin{array}{c}E_{\mathrm{up}} \\
(\mathrm{K})\end{array}$ & $\begin{array}{c}A_{\mathrm{ul}} \\
\left(\mathrm{s}^{-1}\right)\end{array}$ & $\begin{array}{c}\text { Flux (HH212) } \\
\left(\mathrm{Jy} \mathrm{km} \mathrm{s}^{-1}\right)\end{array}$ \\
\hline 1 & 335.089661 & $6_{2-}-6_{1+}$ & 67.4 & $1.65(-4)$ & $0.025 \pm 0.003$ \\
\hline
\end{tabular}

\section{Appendix B: Column density and dust temperature maps}
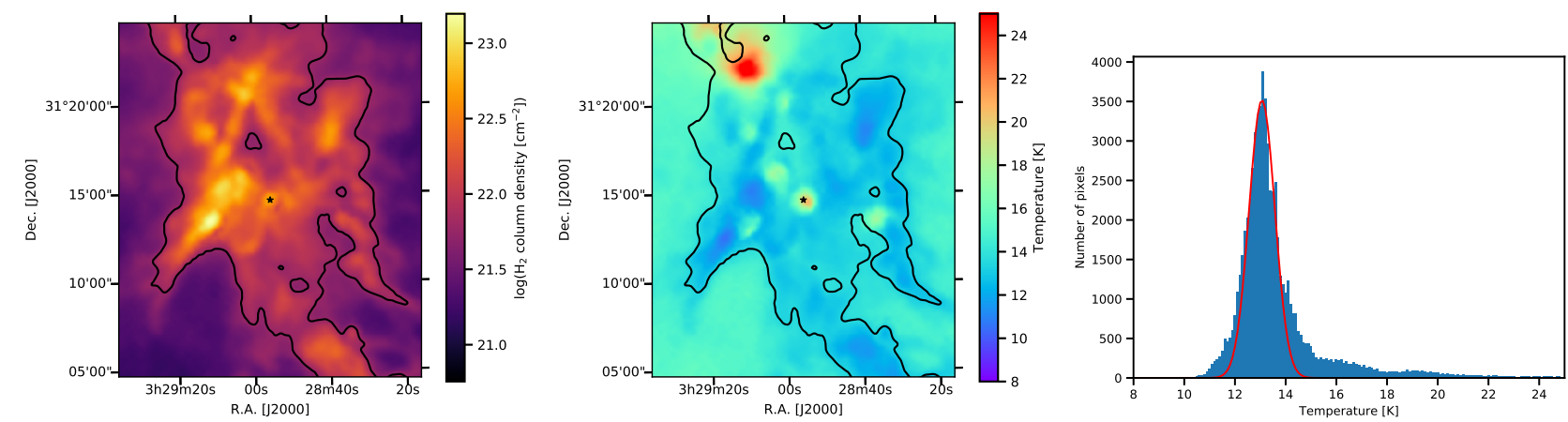

Fig. B.1. $\mathrm{H}_{2}$ column density (left), dust temperature (centre) maps, and dust temperature histogram of the map pixels with $A_{\mathrm{V}}>3$ mag (right) of the NGC 1333 star-forming region surrounding IRAS 2A as observed with the Herschel Space Observatory by the Gould Belt survey (André et al. 2010). The black contour in the maps depicts the 3 mag level. The red curve in the histogram depicts the Gaussian fit of the histogram around the histogram peak. 
Appendix C: Comparison with model predictions
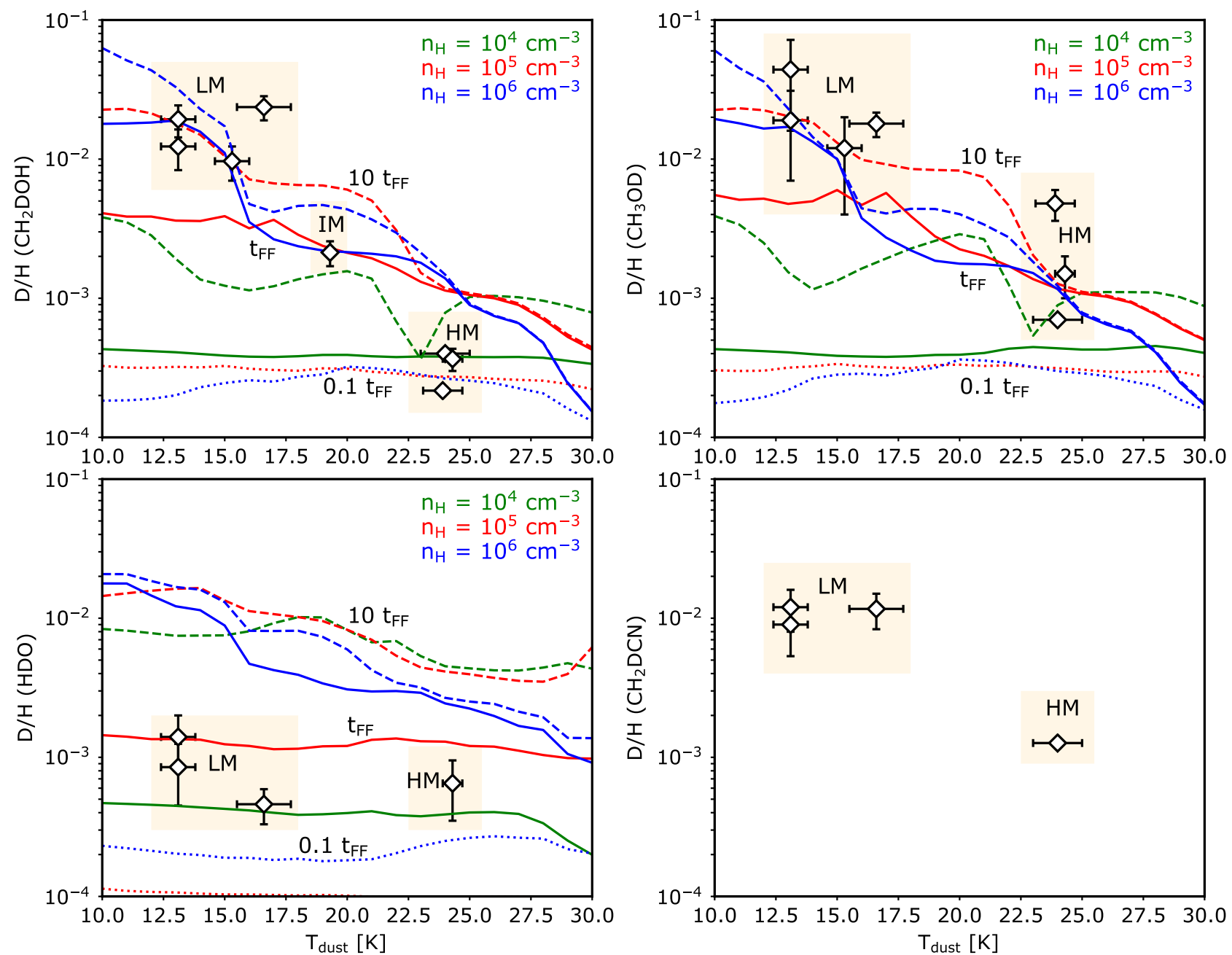

Fig. C.1. Statistical (i.e. taking into account the statistical ratios for the functional groups with multiple $\mathrm{H}$-atoms) $\mathrm{CH}_{2} \mathrm{DOH}\left(\right.$ top left), $\mathrm{CH}_{3} \mathrm{OD}$ (top right), $\mathrm{HDO}$ (bottom left), and $\mathrm{CH}_{2} \mathrm{DCN}$ (bottom right) deuterium fractionations observed towards the hot core of a sample of low-mass, intermediate-mass, and high-mass protostars with sub-millimeter interferometers as a function of the dust temperature of the surrounding cloud measured with the Herschel or Planck telescopes (see values in Table 3). Green, red, and blue curves show the deuterations predicted by the GRAINOBLE astrochemical model in ices as a function of the dust temperature assumed in the dark cloud simulation at $n_{\mathrm{H}}=10^{4}, n_{\mathrm{H}}=10^{5}$ and $10^{6} \mathrm{~cm}^{-3}$, respectively, for at $0.1 \times t_{\mathrm{FF}}$ (dotted lines), $t_{\mathrm{FF}}$ (solid), and $10 \times t_{\mathrm{FF}}$ (dashed), where $t_{\mathrm{FF}}$ is the free-fall time for the corresponding density. No predictions are given for the $\mathrm{CH}_{3} \mathrm{CN}$ deuteration because this molecule is thought to be mostly produced in the vicinity of protostars either on ices or in the gas phase through warmer chemistry. LM, IM, and HM stand for low-mass, intermediate-mass, and high-mass protostars, respectively. See text for more details. 